\title{
Stirring and mixing in two-dimensional divergent flow
}

Article

Published Version

Lukovich, J. V. and Shepherd, T. G. (2005) Stirring and mixing in two-dimensional divergent flow. Journal of the Atmospheric Sciences, 62 (11). pp. 3933-3954. ISSN 1520-0469 doi: https://doi.org/10.1175/JAS3580.1 Available at https://centaur.reading.ac.uk/32103/

It is advisable to refer to the publisher's version if you intend to cite from the work. See Guidance on citing.

To link to this article DOI: http://dx.doi.org/10.1175/JAS3580.1

Publisher: American Meteorological Society

All outputs in CentAUR are protected by Intellectual Property Rights law, including copyright law. Copyright and IPR is retained by the creators or other copyright holders. Terms and conditions for use of this material are defined in the End User Agreement.

\section{www.reading.ac.uk/centaur}

\section{CentAUR}

Central Archive at the University of Reading

Reading's research outputs online 


\title{
Stirring and Mixing in Two-Dimensional Divergent Flow
}

\author{
Jennifer Verlaine Lukovich* And Theodore G. ShePherd \\ Department of Physics, University of Toronto, Toronto, Ontario, Canada
}

(Manuscript received 24 February 2005, in final form 25 April 2005)

\begin{abstract}
While stirring and mixing properties in the stratosphere are reasonably well understood in the context of balanced (slow) dynamics, as is evidenced in numerous studies of chaotic advection, the strongly enhanced presence of high-frequency gravity waves in the mesosphere gives rise to a significant unbalanced (fast) component to the flow. The present investigation analyses result from two idealized shallow-water numerical simulations representative of stratospheric and mesospheric dynamics on a quasi-horizontal isentropic surface. A generalization of the Hua-Klein Eulerian diagnostic to divergent flow reveals that velocity gradients are strongly influenced by the unbalanced component of the flow. The Lagrangian diagnostic of patchiness nevertheless demonstrates the persistence of coherent features in the zonal component of the flow, in contrast to the destruction of coherent features in the meridional component. Single-particle statistics demonstrate $t^{2}$ scaling for both the stratospheric and mesospheric regimes in the case of zonal dispersion, and distinctive scaling laws for the two regimes in the case of meridional dispersion. This is in contrast to two-particle statistics, which in the mesospheric (unbalanced) regime demonstrate a more rapid approach to Richardson's $t^{3}$ law in the case of zonal dispersion and is evidence of enhanced meridional dispersion.
\end{abstract}

\section{Introduction}

Fluid motion in the extratropical stratosphere is dominated by a large-scale, low-frequency, quasihorizontal velocity field. Charney-Drazin filtering (Charney and Drazin 1961) permits propagation of only the planetary-scale Rossby waves into the wintertime stratosphere. Of particular interest is the erosion of the polar vortex as these planetary waves break (Juckes and McIntyre 1987), resulting in filamentation as material is ejected from the polar vortex into the "surf zone," a region of strong mixing at midlatitudes (McIntyre and Palmer 1983; Shepherd 2000). This process of filamentation can be described in terms of chaotic advection, a phenomenon wherein a quasi-regular velocity field gives rise to irregular particle paths (Pierrehumbert 1991; Ngan and Shepherd 1999a; Joseph and

\footnotetext{
* Current affiliation: Centre for Earth Observation Science (CEOS), University of Manitoba, Winnipeg, Manitoba, Canada.
}

Corresponding author address: Dr. T. G. Shepherd, Dept. of Physics, University of Toronto, 60 St. George Street, Toronto, ON, M5S 1A7, Canada.

E-mail: tgs@atmosp.physics.utoronto.ca
Legras 2002). Filamentation has been the subject of much study with regard to the dynamics of a perturbed vortex (e.g., Polvani and Plumb 1992), in part because such an "erosion-entrainment" process sets up conditions for chemical ozone depletion in the springtime polar vortex.

By contrast, the mesosphere is believed to be distinguished by ubiquitous gravity wave activity. Due to decreasing density with increasing altitude, internal inertia-gravity waves grow in amplitude as they propagate upward into the mesospheric region. Although adequate global observational data is not available for the mesosphere, owing to the stringent spatial and temporal resolution requirements necessary to accurately capture mesospheric winds, local measurements suggest the ubiquitous presence of gravity waves (Fritts and Alexander 2003). Moreover, wind fields generated from general circulation models (GCMs) suggest that the mesosphere is characterized by increasing spatial irregularity with altitude (Koshyk et al. 1999). As demonstrated by Shepherd et al. (2000), GCMs also exhibit an increased temporal variability in the mesosphere relative to the stratosphere, as is manifested, for example, in the sensitivity of contour advection calculations to temporal resolution of the winds. 
Differences in stratospheric and mesospheric dynamics are quantified, in part, by kinetic energy spatial wavenumber spectra and their slopes. In terms of an energy spectrum with power-law scaling $E(k) \sim k^{-\beta}$, comparison of flow fields generated from different GCMs demonstrates that the stratosphere is characterized by a steep spectrum with $\beta>3$, with the divergent component much weaker than the rotational component, indicative of balanced (slow, vortical) motion; whereas the mesosphere is distinguished by a much shallower spectrum at smaller spatial (higher wavenumber) scales with $\beta<3$, with the divergent and rotational components of comparable amplitude, indicative of unbalanced (fast) motion (Koshyk et al. 1999). Although these are only GCM results, they are consistent with aircraft measurements of shallow kinetic energy spectra at smaller spatial scales in the lower stratosphere (Bacmeister et al. 1996).

Noteworthy, and key to the present study, are analogous spectral slope properties for what are referred to as (spectrally) nonlocal and local dynamics (Shepherd et al. 2000). For nonlocal dynamics, tracer evolution at a given length scale is governed by velocity features at much longer length scales; for local dynamics, tracer evolution is governed by velocity features at comparable length scales. As outlined by Bennett (1984) for nondivergent (balanced) flow, for a kinetic energy spectrum described by $E(k) \sim k^{-\beta}$, nonlocal dynamics are characterized by $\beta \geq 3$. For $1<\beta<3$, tracer evolution is described by local dynamics, where no single time scale exists. For $\beta \leq 1$, transport is described by diffusion.

Although the above categorization of spectral slopes serves as a means by which the nature of mixing within a given regime may be established, measurements from atmospheric observations or laboratory experiments tend to be, instead, of structure functions and diffusivities. The kinetic energy spectrum is then derived from these measured quantities. The structure function is defined for $\mathbf{u}(\mathbf{x})$, the Eulerian velocity in physical space according to (Babiano et al. 1985)

$$
S(\mathbf{D})=\frac{1}{2}\left\langle|\mathbf{u}(\mathbf{x}+\mathbf{D})-\mathbf{u}(\mathbf{x})|^{2}\right\rangle,
$$

for $\mathbf{x}=(x, y)$ the position vector and $\mathbf{D}=\left(D_{x}, D_{y}\right)$ the separation vector with magnitude $\mathcal{D}$, and where averaging (denoted by the angle brackets) occurs over all Eulerian coordinates. According to Babiano et al. (1985), passive tracer behavior is governed by nonlocal dynamics and thus by the largest spatial scales for $\beta \geq$ 3 . Nonlocal dynamics are then depicted by a structure function scaling law of the form

$$
S(\mathcal{D}) \sim \mathcal{D}^{2} .
$$

Local dynamics, for which $1<\beta<3$, involve length scales comparable to the interparticle separation so that $k \sim \mathcal{D}^{-1}$. The structure-function scaling law is then of the form

$$
S(\mathcal{D}) \sim \mathcal{D}^{\beta-1} .
$$

For the diffusive regime, where $\beta \leq 1$, the structure function is constant with respect to the interparticle separation.

The present work is motivated by the desire to achieve both a qualitative and a quantitative understanding of the effect of an unbalanced component upon the topological and statistical properties of the flow. Of central interest is the influence of the unbalanced component of the velocity field upon the coherent features prevalent in a regime described by chaotic advection. Pertinent questions to be asked in this investigation are how is balanced flow modified with the presence of an unbalanced component? More specifically, how does the unbalanced component affect the velocity field and its spatial gradients, and how are unbalanced dynamics reflected in zonal and meridional dispersion statistics? In addition, what are the implications of this modified flow topology for mixing? This study addresses these questions through the examination of zonal and meridional dispersion statistics. The former provide a framework against which theoretical arguments such as anomalous dispersion and Richardson's (1926) $t^{3}$ law may be tested, in contrast to the latter which, on the sphere, are affected by geometrical constraints. While previous studies have examined (balanced) transport in the context of dispersion along and across potential vorticity (PV) contours (LaCasce and Speer 1999), the present investigation seeks to quantify zonal and meridional dispersion in the stratospheric surf zone, where PV coordinates are not appropriate. Louazel and Hua (2004) have examined the effects of divergent flow on PV evolution, although not on the evolution of an arbitrary tracer, as is considered in the present analysis. The present study seeks also to determine whether diffusion is an appropriate description for meridional transport in the context of mesospheric dynamics. The implications for this investigation reside in the additional question: Is diffusion an appropriate parameterization for transport by unbalanced flow?

To address these questions, we analyze two idealized shallow-water numerical experiments representative of stratospheric and mesospheric dynamics. Section 2 describes the shallow-water model, the experimental setup used to establish a surrogate stratosphere and mesosphere, and the kinetic energy spectra obtained 
from these experiments. Methods and diagnostics used to analyze the experiments, including the generalization of the Hua-Klein criterion to divergent flow, are outlined in section 3. Section 4 presents results from these experiments. Here it is demonstrated that smallscale imbalance significantly influences meridional and, to a lesser extent, zonal dispersion, as highlighted by two-particle (relative) dispersion statistics. Further, the physical implications of unbalanced dynamics for mixing are examined in the context of structure functions, where we find that arguments from two-dimensional (2D) turbulence theory [(2) and (3)] appear insensitive to the introduction of an unbalanced (fast) component to the flow.

\section{The shallow-water model: Balance and imbalance}

Although stratospheric dynamics can be largely described in terms of an essentially nondivergent flow, the presence of gravity waves in the mesosphere does not permit such a description. A dynamical system of equations that incorporates the slowly varying vortical motion in addition to the rapidly varying gravity waves is therefore necessary. The rotating shallow-water equations (SWE) provide such a framework. For a single fluid layer with varying surface height $\eta$ and bottom topography located at $z=-H$, the rotating SWE are given by (e.g., Salmon 1998)

$$
\begin{aligned}
& \frac{D \mathbf{u}}{D t}+f \mathbf{k} \times \mathbf{u}=-g \boldsymbol{\nabla} \eta, \\
& \frac{\partial h}{\partial t}+\boldsymbol{\nabla} \cdot(\mathbf{u} h)=0,
\end{aligned}
$$

where $\mathbf{u}=(u, v)$ is the horizontal velocity, $\boldsymbol{\nabla}=\left(\partial_{x}, \partial_{y}\right)$, $f$ is the Coriolis parameter, $g$ is the gravitational acceleration, $\mathbf{k}$ is the unit vector in the vertical, $D / D t$ is the Lagrangian derivative, and $h=\eta+H$ denotes the depth of the fluid layer. Various investigations (e.g.,
Ngan and Shepherd 1999a,b) have used the SWE to analyze transport in the stratosphere. Here we examine the dynamics resulting from the inclusion of a divergent component in order to analyze transport in a regime characteristic of mesospheric dynamics and contrast this with the stratospheric case.

Analyzed in the present investigation are two shallow-water numerical simulations representative of stratospheric and mesospheric dynamics on a quasihorizontal isentropic surface. The first, referred to as the planetary-wave (PW) experiment, consists of planetary-wave forcing superimposed on a zonal shear flow, and is established to represent dynamics in the wintertime extratropical stratosphere. This setup replicates that of previous studies (e.g., Juckes and McIntyre 1987; Ngan and Shepherd 1999a,b) and uses the same height and mean shear as in the studies of chaotic advection of Ngan and Shepherd (1999a,b). Planetary wave forcing is intended to generate dynamics resulting from Rossby waves in the wintertime stratosphere, which are normally of zonal wave 1 or wave 2 , and thus to reflect the mixing properties of a balanced flow field. The second experiment, referred to as the planetary wave/gravity wave (PW/GW) experiment, consists of forced gravity waves in addition to planetary waves superimposed on the basic state, and is established to represent mesospheric dynamics (albeit in a highly idealized fashion). This is the novel regime and is of interest in itself as an example of strongly divergent flow. It should also be noted that the regime investigated in the present study is that of nonbreaking gravity waves, where the gravity waves provide a high-frequency, small-scale stochastic component to the horizontal velocity field, and particles are confined to isentropic surfaces. For this purpose, a two-dimensional study is appropriate. Clearly, the study is not as relevant to the case of breaking gravity waves, where threedimensional effects would become important.

The additional forcing function for the PW/GW experiment in spectral space is described by

$$
\phi^{\mathrm{GW}}(n, m)= \begin{cases}\phi_{0}^{\mathrm{GW}}\left(\frac{n^{*}\left(n^{*}+1\right)}{n(n+1)}\right)^{1 / 6} \exp i \vartheta(n, m, t), & \text { if } n^{*}<n<N \\ 0, & \text { otherwise. }\end{cases}
$$

Here $\phi \equiv g z$ is the geopotential of the bottom topography located at depth $z=-H, N$ is the model truncation wavenumber, $n$ is the spherical harmonic index, $m$ is the zonal wavenumber, $n^{*}=3$ is the minimum total wavenumber forced for the GW case, and $\phi_{0 \mathrm{GW}}=1.5 \times 10 \mathrm{~m}^{2} \mathrm{~s}^{-2}$ is the amplitude of the gravity wave forcing. Parameters chosen for the PW/GW experiment were found by trial and error to approximately replicate the kinetic energy spectra computed by Koshyk et al. (1999), and analyzed by Shepherd et al. 
(2000), on the isentropic surface $4000 \mathrm{~K}$ (middle mesosphere). The phase $\vartheta(n, m, t)$ is modified temporally via a recursive filter scheme

$$
\vartheta^{\text {new }}(n, m, t)=\gamma \vartheta^{\text {old }}+r\left(1-\gamma^{2}\right)^{1 / 2}
$$

where $\gamma$ defines the temporal autocorrelation $\tau$ of the forcing according to the relation $\tau / \Delta t=1 /(1-\gamma)$, which measures the memory time in terms of the time steps $\Delta t$, and $r$ is a random number found between 0 and $2 \pi$. Here $\gamma=0.92$, corresponding, for model time steps of $\Delta t=15 \mathrm{~min}$, to an $e$-folding time $\tau$ of approximately three hours.

In the statistical analyses, a third experiment consisting of gravity waves with a temporally decorrelated flow field (i.e., the recursive filter scheme implemented for gravity wave forcing is replaced by a random temporal forcing) superimposed on the zonal shear flow, without the PW forcing, is used as a benchmark for Brownian motion, and is referred to as the gravity wave, random in time (GWRT) experiment. The PW and GWRT experiments represent two extremes of balanced and unbalanced flow.

For the current investigation, the model was run with longitude-latitude spatial resolution of $145 \times 72$ points, and sampled every three hours. The geopotential and velocity field are relaxed to the westerly jet (zonal shear flow) on a time scale of ten days. The runs extended for a model time of 70 days, in order to ensure that statistical equilibrium was attained. Subsequent analyses neglect the first 20 days of the run, unless otherwise noted, in order to avoid spurious effects resulting from the initial implementation of the forcing mechanisms.

Shown in Fig. 1 are snapshots of the zonal wind field in the PW and PW/GW experiments illustrating, in addition to the zonal jet, the presence of large-scale coherent features in the PW experiment. In contrast, small-scale features are evident in the PW/GW experiment. Spatial and temporal autocorrelations (not shown) further characterize both the similarity and the distinction between the simulated stratospheric and mesospheric regimes. In particular, similarity in the longitudinal spatial correlation of the geopotential for the two experiments may be attributed to the large-scale features that are set by the planetary-wave forcing mechanism. Differences in temporal behavior are manifested in a much more rapid decorrelation time (on the order of an hour) in the total geopotential for the PW/GW experiment than for the PW experiment. In the latter case, the total geopotential does not appear to decorrelate at all for the time interval examined (over four days for quarter-hour sampling from days 60 a)

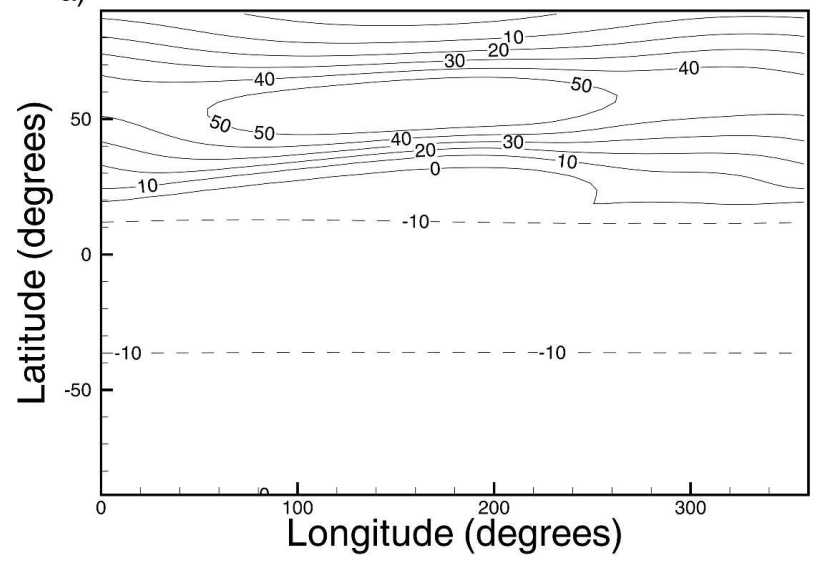

b)

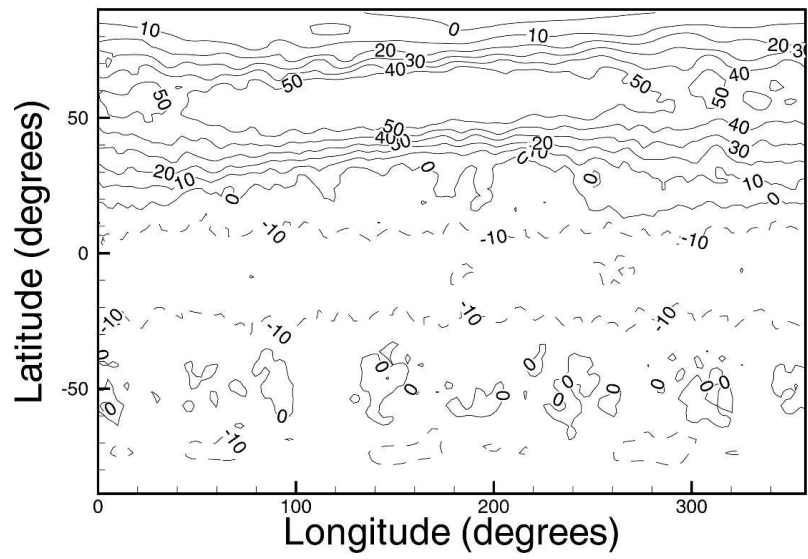

FIG. 1. Instantaneous snapshots of the zonal wind field $\left(\mathrm{m} \mathrm{s}^{-1}\right)$ at $t=70$ days for the (a) $\mathrm{PW}$ and (b) $\mathrm{PW} / \mathrm{GW}$ experiment, with contour intervals of $10 \mathrm{~m} \mathrm{~s}^{-1}$.

to 65), indicative of temporal periodicity in the $\mathrm{PW}$ field. The time scale for the $\mathrm{PW} / \mathrm{GW}$ experiment is the time scale of the gravity waves themselves.

Figure 2 depicts the temporally averaged kinetic energy spectra for the total, rotational and divergent components of the winds for the extratropical Northern Hemisphere. The total-energy power spectrum for the PW experiment exhibits a steep spectral slope, with the rotational component dominating the divergent component. The peak at $n \sim 4$ (length scale $\sim 4000 \mathrm{~km}$ ) indicates energy associated with the large-scale, lowfrequency PWs. For the PW/GW experiment, the steep spectral slope at large spatial scales (small wavenumber) gives way to a shallower spectral slope at smaller scales, where the divergent component is comparable to (in fact, greater than) the rotational component. This is qualitatively consistent with the stratospheric and mesospheric kinetic energy spectra found by Koshyk et al. (1999) in GCMs. 

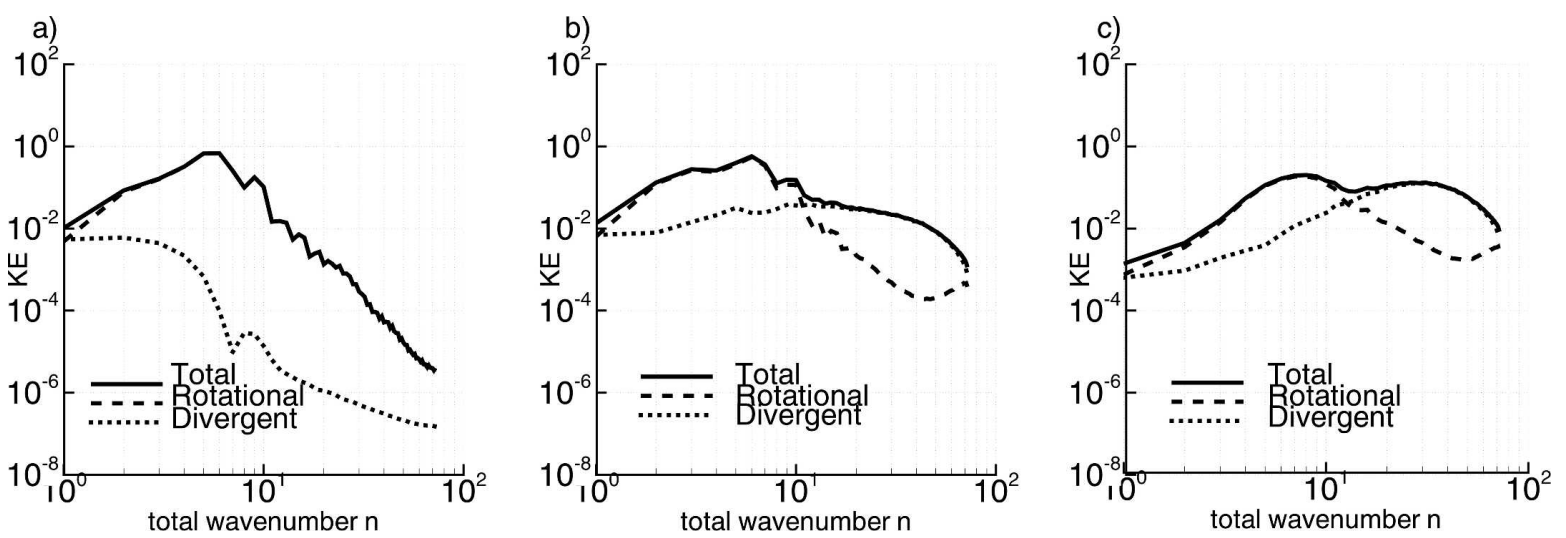

FIG. 2. Time-averaged (20-70 days) kinetic energy spectra $\left(\mathrm{m}^{2} \mathrm{~s}^{-2}\right.$ ) for the (a) PW, (b) PW/GW, and (c) GWRT experiments. Indicated in each panel are the total, rotational, and divergent flow components. In (a) the total and rotational overlap are shown.

Time scales associated with the PW and PW/GW experiments are illustrated in the frequency power spectra for the divergence and the linearized potential vorticity $\omega-h^{\prime} f / h_{*}$ for the PW and PW/GW experiments, where $\omega \equiv \partial_{x} v-\partial_{y} u$ denotes the relative vorticity, $h_{*}$ denotes the mean fluid depth of $8 \mathrm{~km}$, and $h^{\prime}=h-h_{*}$, and are depicted in Fig. 3. Observed in the power spectra for the divergence is an accumulation of power at high frequencies for the $\mathrm{PW} / \mathrm{GW}$ experiment. The power spectra for the linearized potential vorticity indicate an accumulation of power at low frequencies for both the PW and PW/GW experiments, but also significant power at high frequencies for the PW/GW experiment, suggesting a fast component to the linearized potential vorticity in this case.

a)

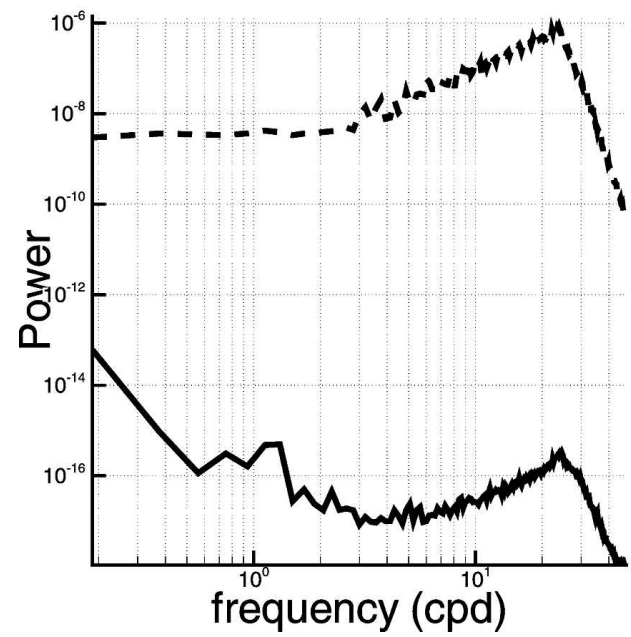

\section{Methods and diagnostics}

\section{a. Geometric analysis: The Hua-Klein criterion and patchiness}

To understand the nature of transport and mixing in these experiments and to determine the influence of the unbalanced component to the flow on the velocity field and its gradients, we characterize geometric aspects of the velocity field using the Eulerian diagnostic of the Hua-Klein criterion and the Lagrangian diagnostic of patchiness.

The behavior of passive tracer motion in twodimensional flow may be elucidated by a partitioning of the flow into vorticity-dominated (elliptic) and straindominated (hyperbolic) components, known as the

\section{b)}

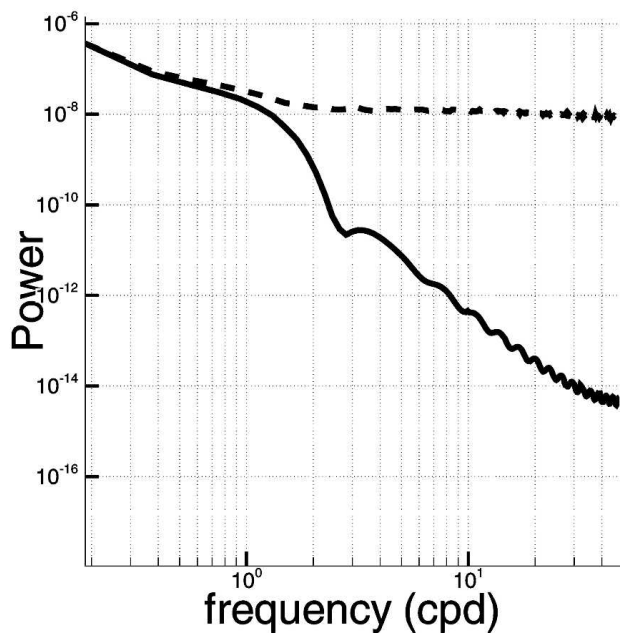

FIG. 3. Power spectra of (a) the divergence $\delta\left(\mathrm{s}^{-2}\right)$ and (b) a linearized and scaled potential vorticity $\omega-h^{\prime} f / h_{*}\left(\mathrm{~s}^{-2}\right)$ for the PW (solid) and PW/GW (dashed) experiments. 
Okubo-Weiss criterion (Okubo 1970; Weiss 1991). This is achieved through consideration of the eigenvalues of the Eulerian velocity gradient tensor, $\partial \mathbf{u} / \partial \mathbf{x}$, determined from the linearized equations of relative motion

$$
\dot{\mathbf{x}}=\frac{\partial \mathbf{u}}{\partial \mathbf{x}} \cdot \mathbf{x},
$$

for $\mathbf{x}$ the (Eulerian) relative position vector, and where the dot denotes the partial time derivative, $\partial / \partial t$. The real component of the square of the eigenvalues of the velocity gradient tensor then yields the expression for the Okubo-Weiss criterion applicable to divergent flow,

$$
Q_{\mathrm{ow}}=\frac{1}{4}\left(\delta^{2}+S^{2}-\omega^{2}+2 \delta \sqrt{S^{2}-\omega^{2}}\right),
$$

where

$$
S^{2}=S_{n}^{2}+S_{s}^{2}
$$

for $S_{n}=\partial_{x} u-\partial_{y} v$ the stretching rate, $S_{s}=\partial_{x} v+\partial_{y} u$ the shear component of the strain, $\omega=\partial_{x} v-\partial_{y} u$ the relative vorticity and $\delta=\partial_{x} u+\partial_{y} v$ the divergence (Provenzale 1999). Regions with $Q_{\text {ow }}>0$ correspond to strong divergence or deformation. Regions with $Q_{\text {ow }}<0$ indicate the dominance of elliptic fixed points.

However, the Okubo-Weiss criterion, in assuming that velocity gradients vary slowly in time with respect to vorticity gradients, has been shown to hold only at the center of vortex cores and near stagnation points (Basdevant and Philipovitch 1994; Hua and Klein 1998). [Lagrangian versions of the Okubo-Weiss criterion, which monitor the finite-time hyperbolicity of a trajectory, relax the conditions of this assumption (Haller 2000; Lapeyre et al. 2001).] In general, the time rate of change in the velocity gradients must be considered. Such is the premise upon which the Hua-Klein criterion (Hua and Klein 1998) is founded. Particle motion is described in terms of the Lagrangian acceleration

$$
\gamma_{L}=\ddot{\mathbf{x}}
$$

The Hua-Klein criterion $Q_{\mathrm{HK}}$ is defined as the largest of the eigenvalues of the acceleration gradient tensor for nondivergent flow. Generalization of $Q_{\mathrm{HK}}$ to encompass divergent flow is determined by substituting (8) into (11), giving

$$
\boldsymbol{\gamma}_{L}=\left(\frac{\partial \dot{\mathbf{u}}}{\partial \mathbf{x}}\right) \cdot \mathbf{x}+\left(\frac{\partial \mathbf{u}}{\partial \mathbf{x}}\right)^{2} \cdot \mathbf{x}
$$

The eigenvalues of the acceleration gradient tensor in the divergent case are hence expressed as

$$
\begin{aligned}
\lambda_{ \pm}= & \frac{1}{4}\left(2 \dot{\delta}+\delta^{2}+S^{2}-\omega^{2}\right) \\
& \pm \frac{1}{2} \sqrt{\begin{array}{l}
\dot{S}_{s}^{2}+\dot{S}_{n}^{2}-\dot{\omega}^{2}+2 \delta\left(\dot{S}_{s} S_{s}+\dot{S}_{n} S_{n}+\dot{\omega} \omega\right) \\
+\delta^{2}\left(S^{2}-\omega^{2}\right) .
\end{array}}
\end{aligned}
$$

The diagnostic implemented to investigate the evolution of the velocity gradients is defined by the largest eigenvalue, $\lambda_{+}$, which we call $Q_{L}$. For nondivergent flow, $Q_{L}=Q_{\mathrm{HK}}$. In the limit of slowly evolving fields, but nonvanishing divergence, $Q_{L}=Q_{\text {ow }}$. Large positive values of $Q_{L}$ correspond to strain-dominated regions and negative values to vorticity-dominated regions. It is this diagnostic that we use as the basis for our Eulerian geometric analysis.

Louazel and Hua (2004) recently developed a generalization of the Hua-Klein criterion to assess the role of fast dynamics on vortex filamentation in a shallowwater model. Their diagnostic differs from ours in that their generalized Hua-Klein criterion is evaluated in strain coordinates and incorporates divergence in the material derivative of the normal and shear strain components. By contrast, our analysis explicitly computes the divergence in the original (unrotated) coordinate system.

Geometrical structure may also be assessed using the diagnostic of patchiness or the representation of distinct average velocities in the flow (Pasmanter 1988; Malhotra et al. 1998). For a given fluid particle trajectory, the Lagrangian mean velocity measured along the particle path is expressed (in the notation of Malhotra et al. 1998) as

$$
v_{x}=\frac{[x(t)-x(0)]}{t}, \quad v_{y}=\frac{[y(t)-y(0)]}{t}
$$

for an interval of time $t$. While the Hua-Klein criterion examines kinematic features associated with the geometric properties of the flow, patchiness may be used to analyze its dynamical properties, through investigation of the evolution of the average Lagrangian velocity. It has been shown (Malhotra et al. 1998) that patchiness is useful for the detection of large-scale inhomogeneities in the flow.

\section{b. Statistical analysis}

In this investigation we seek also to determine the influence of unbalanced flow on zonal and meridional dispersion statistics. Complementary to the geometrical 
assessment performed using the Hua-Klein and patchiness diagnostics is a statistical assessment based on single-particle (absolute) and two-particle (relative) dispersion. Absolute dispersion monitors the translation of a single particle (Taylor 1921) and is defined as (in the notation of Provenzale 1999)

$$
\begin{aligned}
A^{2} & =\left\langle\left|x_{i}(t)-x_{i}(0)-\left\langle x_{i}(t)-x_{i}(0)\right\rangle\right|^{2}\right\rangle \\
& =\frac{1}{N} \sum_{i=1}^{N}\left|x_{i}(t)-x_{i}(0)-\left\langle x_{i}(t)-x_{i}(0)\right\rangle\right|^{2},
\end{aligned}
$$

where $x_{i}(t)$ denotes the position of the $i$ th particle at time $t$, and $N$ is the number of particles in the ensemble. Angle brackets denote averages over the particle ensemble, and dispersion can be defined in both the zonal and meridional directions.

Of particular interest in describing the properties of a flow using single-particle dispersion is the scaling behavior

$$
A^{2} \sim t^{\alpha}
$$

when $\alpha \neq 1$ and $\alpha \neq 2$. Anomalous diffusion refers to the phenomenon wherein long-time, large-scale tracer transport exhibits behavior that departs from the expected diffusive limit $\alpha=1$. Ballistic dispersion $(\alpha=2)$ is a special case of superdiffusion $(\alpha>1)$, which is distinguished by a mean-square displacement that grows more rapidly than linearly with time and is a signature of long-range correlations in the Lagrangian velocity, corresponding to large-scale (and lowfrequency) inhomogeneities in the Eulerian velocity field. In contrast, subdiffusive behavior $(\alpha<1)$ is distinguished by a mean-square displacement that grows more slowly than linearly with time and is characteristic of trapping events, which would occur if particles followed regular orbits and experienced a coherent, temporally periodic velocity field.

It is also of interest to note the link between absolute dispersion and the Lagrangian velocity autocorrelation. In the case of statistically stationary flow, the Lagrangian integral (decorrelation) time scale $T_{L}$ is defined as (Majda and Kramer 1999)

$$
T_{L}=\int_{0}^{\infty} R(\tau) d \tau,
$$

where

$$
R(\tau)=\frac{\langle V(t) V(t+\tau)\rangle}{\left\langle V^{2}(t)\right\rangle}
$$

is the Lagrangian autocorrelation, and $V$ is the departure of the Lagrangian velocity from the ensemble av- erage. For sufficiently short times, $t \ll T_{L}$, where $t$ is the elapsed time, the particles have memory of their initial velocities, and temporal dispersion is described by the ballistic regime. By contrast, for very long times, $t \gg T_{L}$ (assuming that $T_{L}<\infty$ ), the particles lose memory of their initial velocities and follow independent random walks as in Brownian motion so that the diffusive limit is attained. When statistical stationarity cannot be assumed, (17) is also a function of time so that a temporal average of the ensemble is required. Nevertheless, a qualitative comparison between autocorrelation and dispersion behavior may still be used to compare the statistical properties of balanced and unbalanced flow.

The statistical analyses presented in section 4 are based on an ensemble of 21824 particles, initially aligned along latitudes separated by $1.0^{\circ}$ and with a longitudinal spacing of $0.5^{\circ}$. The particles are initially confined to the domain bounded by latitudes $15.5^{\circ}$ and $52.5^{\circ}$. Tracer trajectories and calculations of zonal dispersion allow for wrapping past $360^{\circ}$ longitude. All absolute and relative dispersion probability distribution functions (PDFs) are normalized to have unit area. The advection is performed using a particle advection scheme developed by K. Ngan (see Ngan and Shepherd 1999a), with a time step of three hours, corresponding to the model wind sampling interval.

The PDF of particle displacements provides a measure of the evolution of a passive scalar. Departures from Gaussianity reflect the presence of long-range correlations. The existence of long-range correlations in particle transport is manifested in the PDF skewness

$$
S=\frac{\left\langle X^{3}\right\rangle}{\sigma^{3}},
$$

and the flatness

$$
F=\frac{\left\langle X^{4}\right\rangle}{\sigma^{4}}
$$

where $\sigma=\sqrt{A^{2}}$ is the standard deviation of the single particle displacement, and $X=x(t)-x(0)-\langle x(\mathrm{t})-$ $x(0)\rangle$. Skewness is a measure of asymmetry in the PDF and is zero in the Gaussian limit; flatness monitors distribution broadness, and $F=3$ for Gaussian PDFs. Broader-than-Gaussian tails with $F>3$ indicate longrange correlations in the Lagrangian velocity that, in turn, give rise to large particle displacements. As the advected fluid particles encounter coherent structures, dispersion by Brownian motion, expected for homogeneous turbulence, is replaced by anomalous transport evidenced in non-Gaussian PDFs.

While absolute dispersion monitors the translation of a single particle, relative dispersion monitors the sepa- 
ration between a pair of particles. The concept of relative dispersion was introduced by Richardson (1926) and is defined as

$$
D^{2}\left(t ; t_{0} ; \mathbf{D}_{0}\right)=\frac{1}{N} \sum_{i=1}^{N}\left|x_{i}(t)-x_{i+1}(t)-\left\langle x_{i}(t)-x_{i+1}(t)\right\rangle\right|^{2},
$$

for $\mathbf{D}_{0}$ the initial separation between a pair of particles $x_{i}$ and $x_{i+1}, N$ the number of pairs with initial separation $\mathbf{D}_{0}$, and where angle brackets again denote an ensemble average. Here, as in (15), dispersion can be defined in both the zonal and meridional directions. [It should be noted that in the relative dispersion analysis, the magnitude of separation between particle pairs is monitored so that $\left\langle x_{i}(t)-x_{i+1}(t)\right\rangle=0$, where angle brackets denote an ensemble average, in order to guarantee symmetry in labeling.] The separation vector is hereinafter denoted as $\mathbf{D}=\left(D_{x}, D_{y}\right)$ with magnitude $\mathcal{D}$. In monitoring the relative motion between a pair of particles, relative dispersion incorporates the effects of varying sized eddies upon a cluster of particles.

Relative dispersion monitors the straining mechanisms acting on scales comparable to the interparticle separation and is thus dependent on velocity gradients, rather than expressly on the velocity field itself as is single-particle dispersion (Batchelor 1951). This suggests that the correlations of the Lagrangian velocity gradients, instead of the Lagrangian velocities themselves, provide a link between relative dispersion and temporal autocorrelations. One would therefore expect the Eulerian diagnostics of $Q_{\mathrm{Ow}}$ and $Q_{L}$, which monitor the combined effect of velocity gradients, to be reflected in the relative dispersion statistics. Moreover, the time evolution of the separation of a particle pair reflects the accelerating process of relative dispersion (Babiano et al. 1990). This is demonstrated by the differential equation for the separation (Babiano et al. 1990)

$$
\frac{d}{d t}\left(\frac{\left|\mathbf{D}-\mathbf{D}_{0}\right|^{2}}{t}\right)=\left|\mathbf{W}\left(t, \mathbf{D}_{0}\right)\right|^{2}-\left|\frac{1}{t} \int_{0}^{t} \tau \frac{d \mathbf{W}(\tau)}{d \tau} d \tau\right|^{2},
$$

where $\mathbf{W}=\left(W_{x}, W_{y}\right)$ is the relative Lagrangian velocity associated with the relative separation vector $\mathbf{D}$. In particular, the inclusion of the acceleration term in the expression for the time evolution in the separation between a pair of particles is reminiscent of the HuaKlein criterion, and incorporates a sensitivity to unbalanced motion. For the short-time limit $t \rightarrow 0$, the zonal relative acceleration is constant as the particles undergo linear displacement so that the zonal relative dispersion satisfies

$$
D_{x}^{2}=D_{0 x}^{2}+2 D_{0 x} \cdot\left\langle W_{x}(t)\right\rangle t+2 s_{x}\left(D_{0 x}\right) t^{2},
$$

where $s_{x}\left(D_{0 x}\right)=\left\langle\left|W_{x}\left(D_{0 x}\right)\right|^{2}\right\rangle / 2$ is the second-order, one-dimensional structure function. In the long-time limit $t \rightarrow \infty$, relative dispersion is characterized by diffusion, as particle pairs lose memory of their origin, so that $D^{2} \sim t$. Richardson's (1926) $t^{3}$ law holds for intermediate times under the assumption of statistical stationarity in the Lagrangian relative accelerations (as is noted in Zouari and Babiano 1994), and was initially derived by Richardson from observations under the assumption of a separation-dependent diffusivity.

\section{c. Structure functions: Spectral nonlocality and locality}

The implications of modified flow topology resulting from unbalanced dynamics for mixing are addressed using the concepts of spectral nonlocality and locality. Spectrally local and nonlocal properties of stratospheric and mesospheric dynamics, as determined from the PW and $\mathrm{PW} / \mathrm{GW}$ experiments, respectively, may be quantified using the scaling laws derived from the structure functions outlined in the introduction.

The structure functions presented in section 4 are computed from the winds according to (1), for displacements in the zonal direction only. It is assumed that due to similarities in the zonal and total dispersion resulting from the zonal shear flow, structure functions in the zonal direction would provide a valid interpretation of spectrally nonlocal and local behavior for the total (zonal and meridional) flow. Averaging for this purpose occurs over time and over all longitudes at $45^{\circ} \mathrm{N}$.

\section{d. Eddy diffusivities}

Eddy diffusivities can be used in the development of tracer mixing parameterization schemes that model unbalanced flow, as determined from the aforementioned statistical analysis. Passive tracer behavior for a tracer $\mathcal{T}$ is governed by the advection-diffusion equation

$$
\frac{\partial\langle\mathcal{T}\rangle}{\partial t}+\mathbf{V} \cdot \boldsymbol{\nabla}\langle\mathcal{T}\rangle=\boldsymbol{\nabla} \cdot(K \cdot \nabla\langle\mathcal{T}\rangle)
$$

where $K$ refers to an effective eddy diffusivity, or the sum of the molecular diffusivity $k$ and a turbulent eddy diffusivity $K_{T}$ resulting from fluctuations in the advecting velocity $\mathbf{v}=\mathbf{V}+\mathbf{v}^{\prime}$, for $\mathbf{V}$ the mean advective velocity and $\mathbf{v}^{\prime}$ the random, turbulent fluctuations. It should be noted that (24) is not a closed equation: the process of averaging introduces a new variable $K_{T}$, via $\left\langle\mathbf{v}^{\prime} \cdot \boldsymbol{\nabla} \mathcal{T}\right\rangle=-\boldsymbol{\nabla} \cdot\left(K_{T} \cdot \nabla\langle\mathcal{T}\rangle\right)$ (Majda and Kramer 1999). 
It is the closure problem that is responsible for the ambiguity inherent in defining a generalized advectiondiffusion equation and, as a consequence, an eddy diffusivity.

Pertinent to the present discussion is the relationship between the eddy diffusivity and single-particle dispersion (Batchelor 1949). The absolute eddy diffusivity is defined such that (Babiano et al. 1990)

$$
K_{A} \equiv \frac{1}{2} \frac{d A^{2}}{d t},
$$

where $A^{2}$ denotes the absolute dispersion as defined in (15).

Richardson (1926) addressed the question of the presence of a wide range of eddy diffusivities from atmospheric measurements. Richardson's study was motivated by a desire to determine a generalized eddy diffusivity, independent of particle location, but dependent on particle separation. Richardson noted that a constant diffusivity provides an incomplete description of atmospheric dispersion in the presence of eddies, as is evidenced in the expression for the relative diffusivity $K_{R}$, or diffusivity of neighbors (Richardson 1926)

$$
K_{R}=\varepsilon \mathcal{D}^{4 / 3},
$$

where $\varepsilon$ is a constant independent of relative separation, obtained from empirical arguments and observations.

The analytic expression for the relative diffusivity, or time evolution in the separation variance, underlines the role of relative dispersion in addressing the range of eddy diffusivity values in the atmosphere. The relative diffusivity is defined, in a manner akin to the definition for the absolute eddy diffusivity (25), as (Babiano et al. 1990)

$$
K_{R}\left(t, \mathbf{D}_{0}\right) \equiv \frac{1}{2} \frac{d\langle\mathbf{D} \cdot \mathbf{D}\rangle}{d t}=\frac{1}{2} \frac{d D^{2}\left(t, \mathbf{D}_{0}\right)}{d t},
$$

for $D^{2}=\langle\mathbf{D} \cdot \mathbf{D}\rangle$, and where the arguments $\mathbf{D}_{0}$ and $t$ have been introduced to emphasize the dependence of the eddy diffusivity on the initial interparticle separation $\mathbf{D}_{0}$ and on time $t$. Thus, in contrast to the absolute diffusivity $K_{A}$, the relative diffusivity need not be constant in time in order to be well defined. In fact, the significance of the relative diffusivity rests on its ability to demonstrate that the rate of diffusion increases with interparticle separation (Richardson 1926).

It is also possible to determine an (instantaneous) relative diffusivity, defined, in contrast to (27), as

$$
K_{i}=\frac{1}{2}\left[\left\langle\left(\frac{d}{d t} \mathbf{D} \cdot \mathbf{D}\right)^{2}\right\rangle\right]^{1 / 2},
$$

to be computed from the expression (Babiano et al. 1985)

$$
K_{i}=\mathcal{D} S_{\|}^{1 / 2}(\mathcal{D})
$$

where here the longitudinal structure function $S_{\|}(\mathcal{D})$ is now dependent on $\mathcal{D}$ the interparticle separation based on a conditional ensemble averaged where $|\mathbf{D}|=D$, independent of the initial interparticle separation. This allows for the diffusivity to be specified in a diffusion parameterization scheme based on an appropriate model grid size.

\section{Numerical results}

\section{a. Geometric analysis}

The role of unbalanced dynamics in modifying flow topology is shown in the Okubo-Weiss $\left(Q_{\mathrm{ow}}\right)$ and generalized Hua-Klein $\left(Q_{L}\right)$ fields for the $\mathrm{PW}$ and $\mathrm{PW} /$ GW experiments (Fig. 4). Noteworthy is the erosion of large-scale spatial gradients with the inclusion of an unbalanced component to the flow, evidenced in the destruction of the strain-dominated region for the PW/ GW as compared to the PW case. This indicates that velocity gradients are indeed modified by fast dynamics.

For the PW experiment $Q_{\text {ow }}$ is rather similar (though certainly not identical) to $Q_{L}$. This suggests that the velocity gradients are slowly varying in time with respect to the vorticity gradients, which in turn indicates that the changes in the rotational component of the flow, associated with balanced (slow) dynamics, dominate. Moreover, the similarity in $Q_{\mathrm{OW}}$ and $Q_{L}$ in spite of the time-dependent terms evident in the latter implies the existence of a single advective time scale imposed by the planetary waves superposed on the basic-state shear. This is suggestive of nonlocal dynamics, as described in the introduction.

A much more marked contrast between the $Q_{\mathrm{Ow}}$ and $Q_{L}$ fields is found for the PW/GW experiment, as demonstrated in the order of magnitude increase in $Q_{L}$ relative to $Q_{\text {ow }}$, and as is to be expected owing to the large unbalanced component associated with the highfrequency gravity waves. Hence the strain and divergent components evolve more rapidly than for balanced flow so that the significant gradients in vorticity found for the PW experiment are replaced by comparably and more rapidly evolving gradients in the strain and divergent components. This dissimilarity highlights the existence of a rapidly evolving (gravity wave) field that introduces numerous time scales for the PW/GW case and implies spectrally local dynamics. 
a)

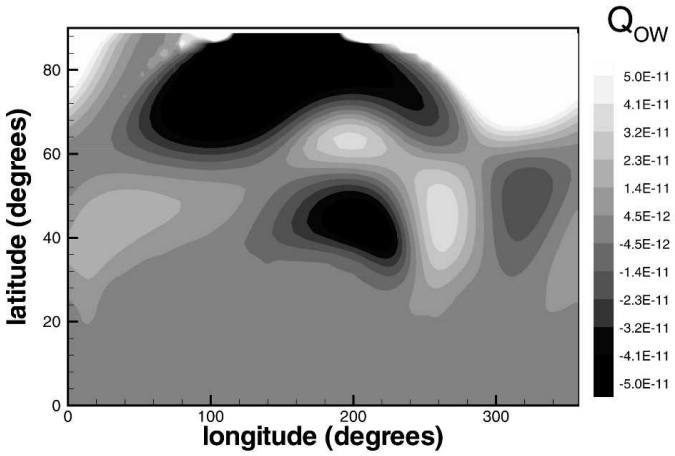

c)

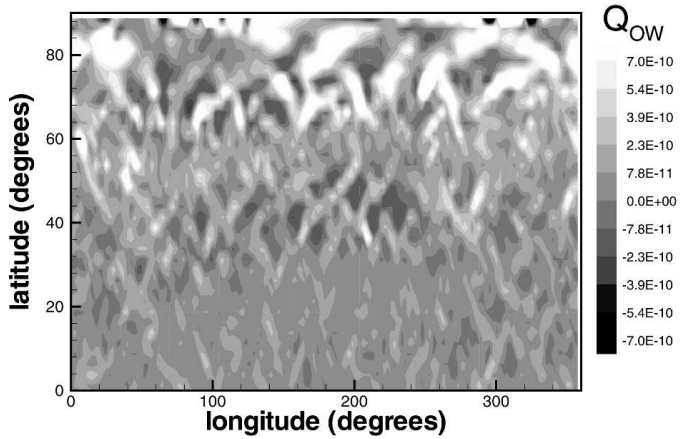

b)

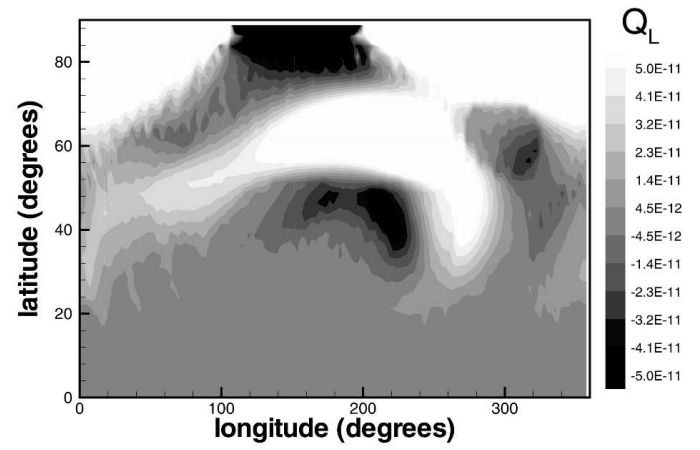

d)

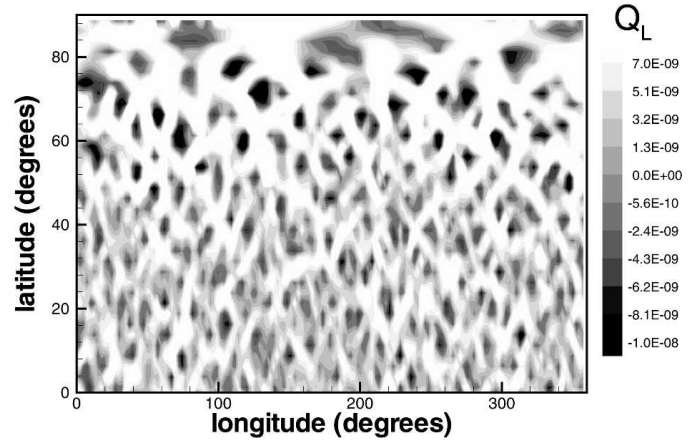

FIG. 4. Grayscale plots determined from the Okubo-Weiss criterion, $Q_{\mathrm{OW}}$, for the (a) PW and (c) PW/GW experiments, and from the generalized Hua-Klein criterion with a divergent component, $Q_{L}$, for the (b) PW and (d) $\mathrm{PW} / \mathrm{GW}$ experiments, all at $t=45$ days. Units are in $\mathrm{s}^{-2}$. Note the difference in scale between (c) and (d).

Shown in Fig. 5 are the patchiness plots for the zonal winds in the $\mathrm{PW}$ and $\mathrm{PW} / \mathrm{GW}$ experiments at $t=0,10$, and 20 days. The initial distribution (Figs. 5a and 5d) corresponds to the Eulerian zonal velocity field. Evident in Fig. 5a is the wave-1 cat's eye structure resulting from the PW forcing. Regions of maximal patchiness at $t=0$ coincide with maximum instantaneous zonal velocities. The dominance of the shear flow in the zonal direction gives rise to large zonal particle displacements, as shown by the maximum values at the core of the zonal jet. Moreover, dominant coherent features remain largely intact for the $\mathrm{PW} / \mathrm{GW}$ experiment, as is demonstrated in Figs. 5d-f. In fact, there is an organization and intensification of the structure as time proceeds and the integrated effect of the small-scale features averages out, leaving the effect of the lowfrequency, large-scale structure.

The variability found at the edge of the eastward jet for the PW/GW experiment suggests that some mixing occurs at the periphery of the jet because trajectories with different Lagrangian histories are contributing to the structure. Enhanced transport into and out of the cat's eye structure is apparent at $t=0$, where the cat's eye takes on a more diffusive character for the PW/GW experiment and, as previously noted, at later time organizes to leave a large-scale, low-frequency structure similar to the PW case. The erosion of patches is consistent with the behavior predicted by Pasmanter (1988) in considering the effects of turbulent diffusion in the presence of coherent structures, and with the effects of inclusion of noise as examined by Malhotra et al. (1998). Larger patches are relatively insensitive to the spatially random and high-frequency fluctuations: it is only the smaller patches that disappear. While the variability in patchiness geometry with the gravity waves might be thought to imply increased transport and perhaps mixing, the persistence of the coherent features in the zonal direction discards such a proposition. Dispersion in the direction of the zonal shear flow would appear to be largely unaffected by the presence of the gravity waves.

This behavior is in contrast to transport transverse to the zonal shear flow. Whereas transport in the zonal direction is governed by the combined effects of the basic-state shear flow, planetary-wave and gravity wave forcing, transport in the meridional direction is gov- 
a)

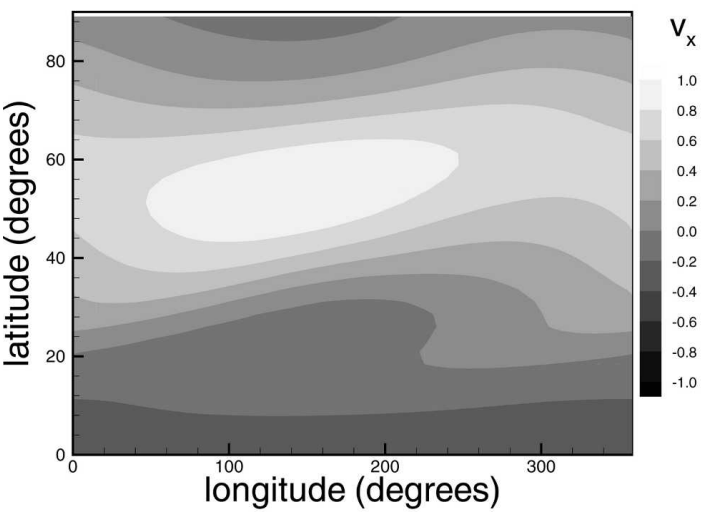

b)

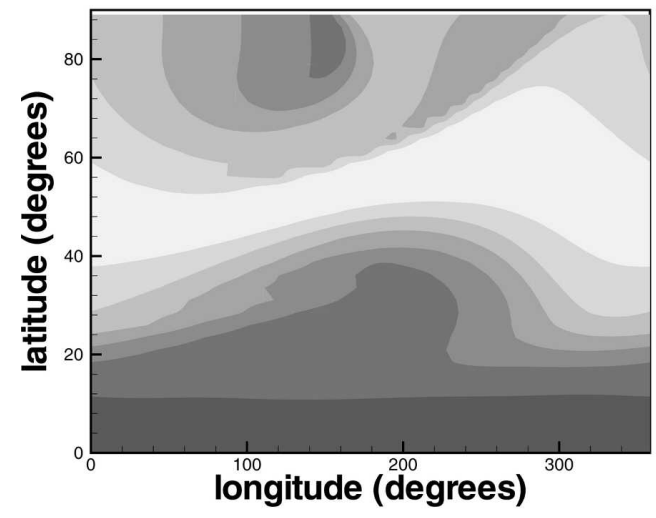

c)

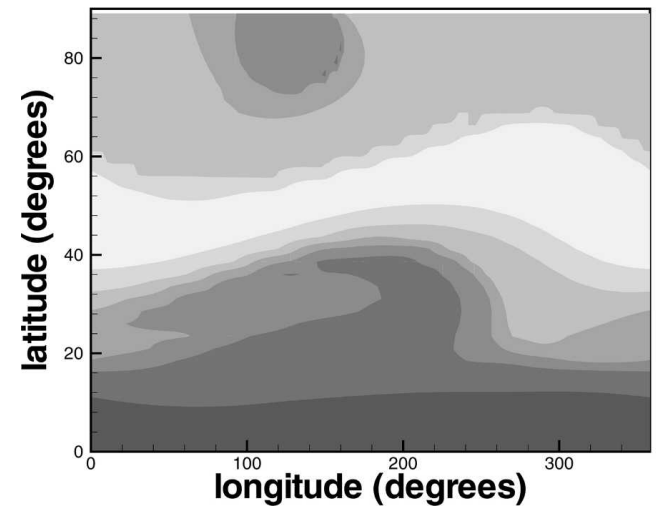

d)

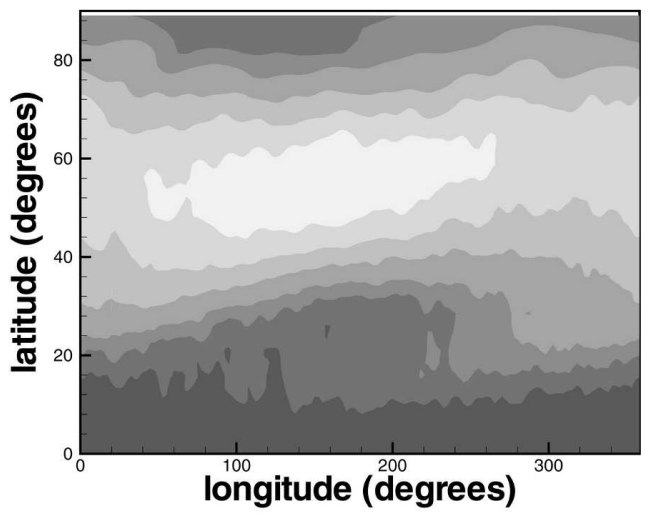

e)

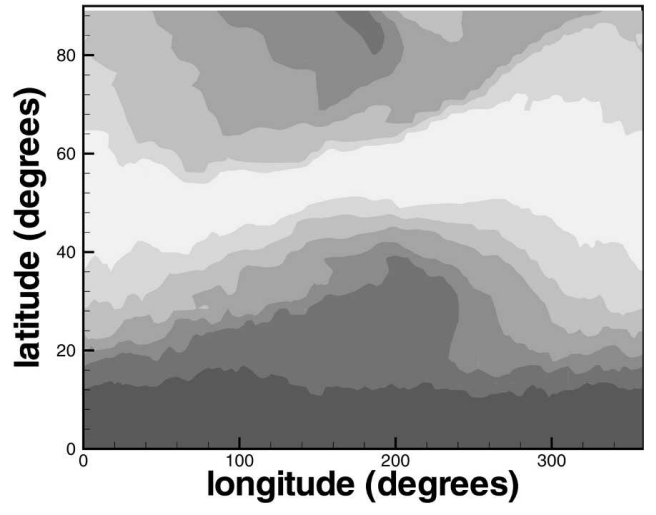

f)

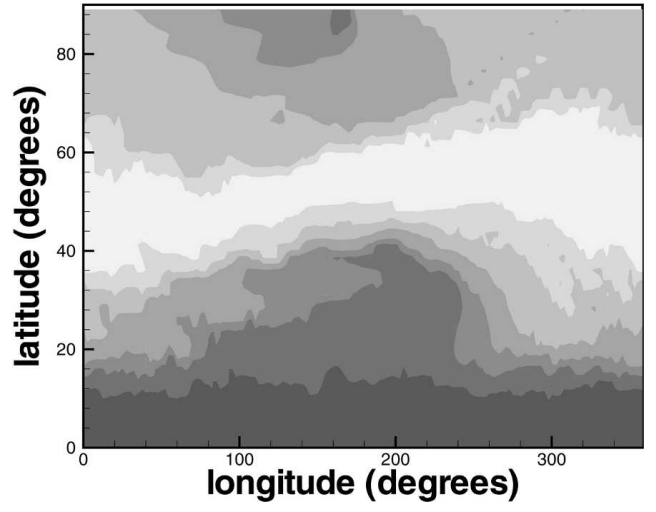

FIG. 5. Patchiness plots [as a function of $\mathbf{x}(0)$ ] for zonal winds (normalized) for the PW experiment at (a) $t=0$ days, (b) $t=10$ days, and (c) $t=20$ days, and for the PW/GW experiment at (d) $t=0$ days, (e) $t=10$ days, and (f) $t=20$ days. Maximal (minimal) patchiness denoted by light (dark) shading.

erned only by the planetary wave and gravity wave forcing. This is emphasized in the patchiness plots for meridional winds. Figure 6 shows that the initial patchiness plot for the $\mathrm{PW} / \mathrm{GW}$ experiment is more diffusive in character than at $t=10$ days. Smaller patches merge to produce the larger patch at midlatitudes, which begins to disintegrate at $t=20$ days. We speculate that the introduction of an initially random field to the meridional flow generates motion akin to Brownian motion: if the gravity waves give a random walk on top of the (spatially smooth) large-scale velocity field, then their effect on $v_{y}$ rapidly goes like $\sqrt{t}$, and hence becomes negligible compared to the contribution to $v_{y}$ from the large-scale motion, which goes like $t$. Consequently, the 
a)

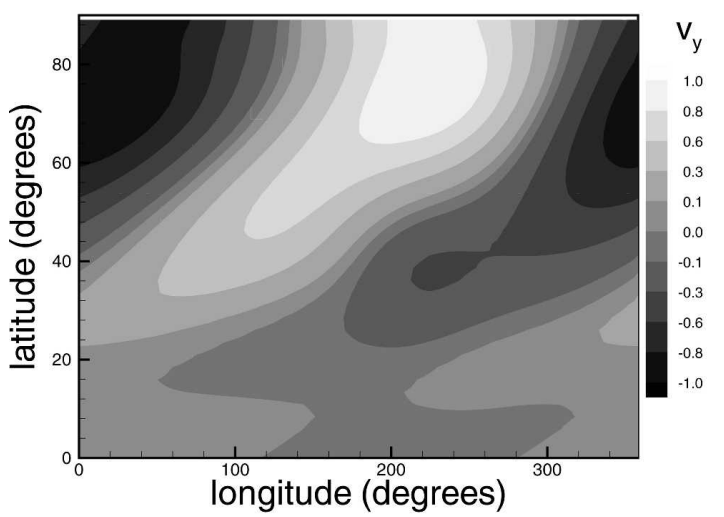

b)

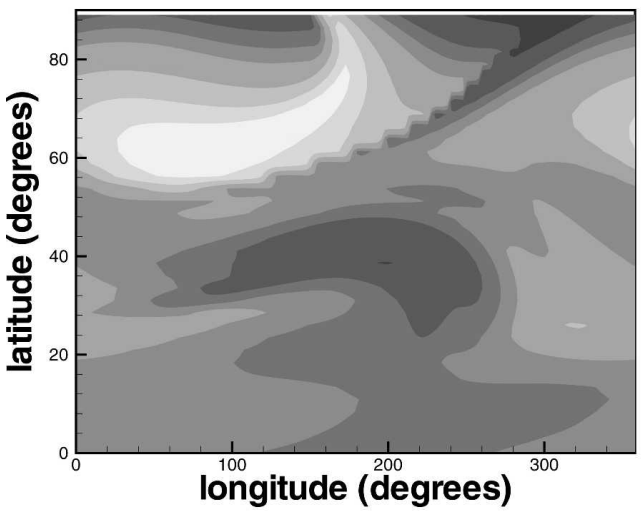

c)

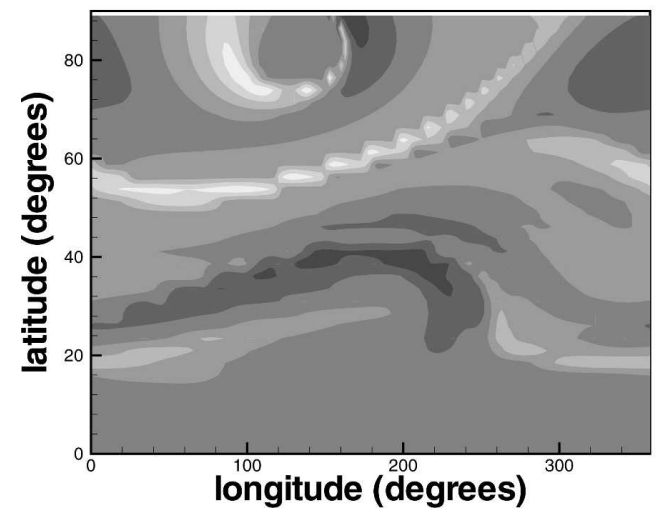

d)

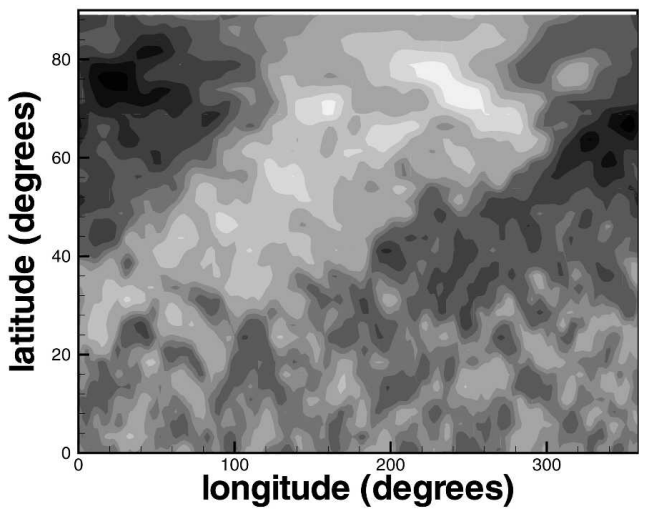

e)

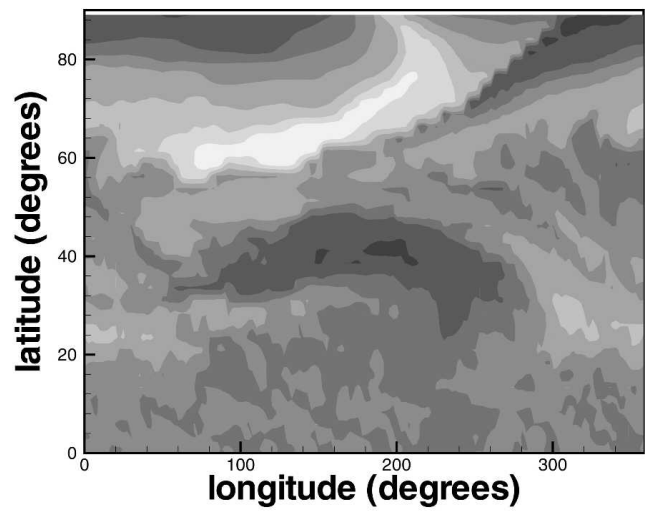

f)

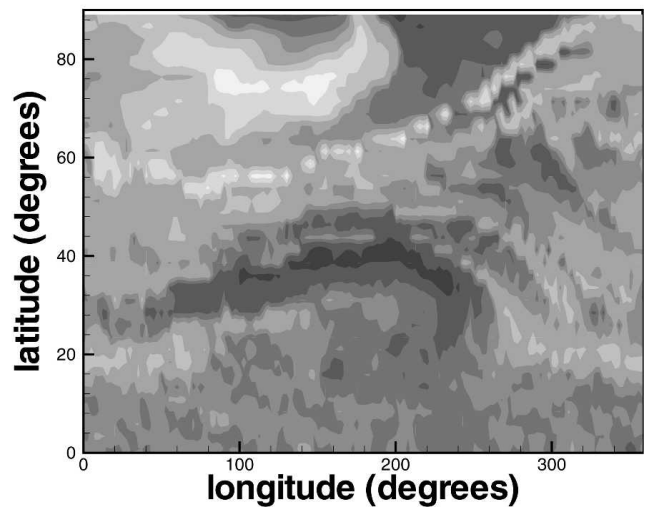

FIG. 6. Patchiness plots [as a function of $\mathbf{x}(0)$ ] for meridional zonal winds (normalized) generated for the PW experiment at (a) $t=0$ days, (b) $t=10$ days, and (c) $t=20$ days, and for the $\mathrm{PW} / \mathrm{GW}$ experiment at (d) $t=0$ days, (e) $t=10$ days, and (f) $t=20$ days. Shading as in Fig. 5 .

patchiness "organizes" until the large-scale motion itself breaks up (after 20 days). Noteworthy also is enhanced variability in the surf zone for the PW/GW experiment relative to the PW experiment, demonstrating an increased possibility for particles to escape the midlatitude region of strong mixing, and reminiscent of the significant erosion of the velocity gradients observed for both the $Q_{\text {ow }}$ and $Q_{L}$ fields in Fig. 4. Unbalanced dynamics, as manifested in gravity wave activity, are therefore shown to provide a significant contribution to dispersion in the meridional direction, given the absence of mean meridional flow. These patchiness results are further illustrated in the single-particle dispersion results. 
(a)

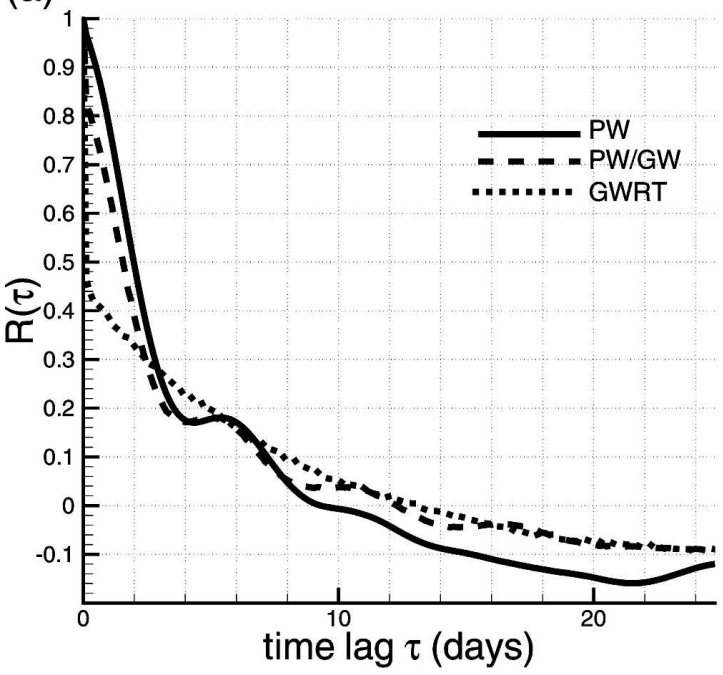

(b)

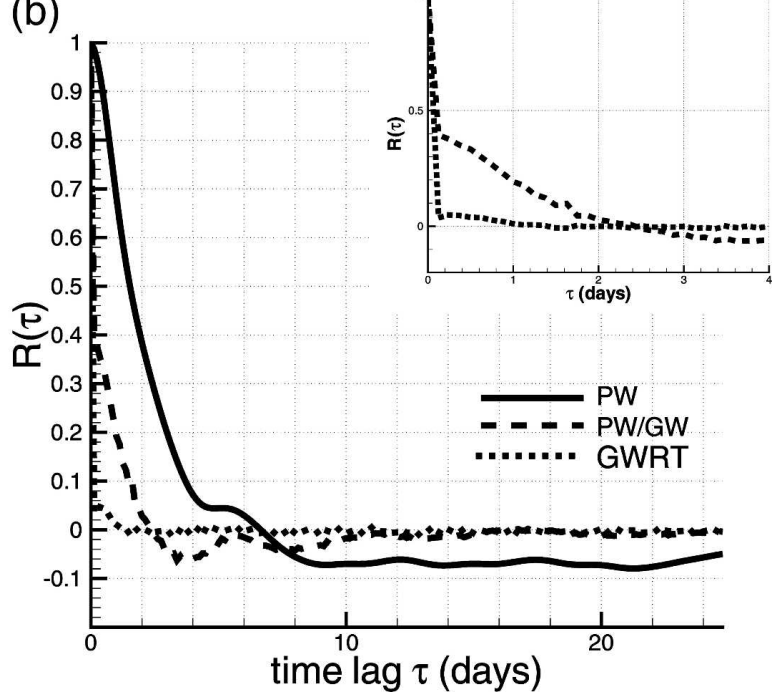

FIG. 7. Ensemble-averaged Lagrangian autocorrelations for (a) zonal and (b) meridional winds, averaged over all longitudes $\left(15.5^{\circ}-52.5^{\circ} \mathrm{N}\right)$. The inset in (b) shows a blow up of the PW/GW and GWRT curves for the first few days.

\section{b. Statistical analysis}

The effect of unbalanced dynamics on zonal and meridional dispersion statistics is captured in the ensemble-averaged Lagrangian autocorrelations for the zonal and meridional winds, computed for the $\mathrm{PW}, \mathrm{PW} /$ GW, and GWRT experiments (Fig. 7). An ensemble of 700 particles was used in contrast to the 21824 particles used in other statistical analyses performed here, due to memory constraints. Evident in Fig. 7a is the absence of a decorrelation time for the zonal wind: the presence of the zonal jet in the form of a basic-state shear flow establishes long-range correlations in the flow in all three experiments. Anticorrelation in the winds over long time scales may be a consequence of meridional particle migration, or a signature of oscillatory behavior. The experiments would need to be run longer to confirm the latter. While one cannot accurately estimate $R(\tau)$ because of nonstationarity, one would nevertheless expect, from the long-range correlations established by the zonal shear flow in all three experiments, zonal dispersion to be characterized by superdiffusion.

Indeed, ballistic behavior is observed in the ensemble-averaged absolute zonal dispersion for the PW, PW/GW, and GWRT experiments, depicted in Fig. 8. The similarity in the behavior of the three experiments suggests that single-particle dispersion is insensitive to the behavior of small-scale eddies. Neither the smallerscale gravity waves resulting from the forcing at higher wavenumbers, or even the planetary wave, are reflected in the absolute zonal dispersion. Rather, the large-scale sweeping effects exerted by the westerly jet dominate. These results are consistent with results found for hori- zontal transport in a meandering jet (Samelson 1992). It is also of interest to note that for mean-shear-controlled dispersion, the relationship $A^{2} \sim \partial_{y} u^{2} t^{2}$ is satisfied in the case of zonal dispersion. In particular, for a typical value of $-2.0 \times 10^{-11} \mathrm{~s}^{-2}$ for $Q_{O W}$ (see the rotationdominated region in Fig. 4 a near $50^{\circ} \mathrm{N}$-as is evident in the large gradients across the zonal shear flow shown in Fig. 5, the vorticity is dominated by $\left.\partial_{y} u\right), \partial_{y} u \sim$

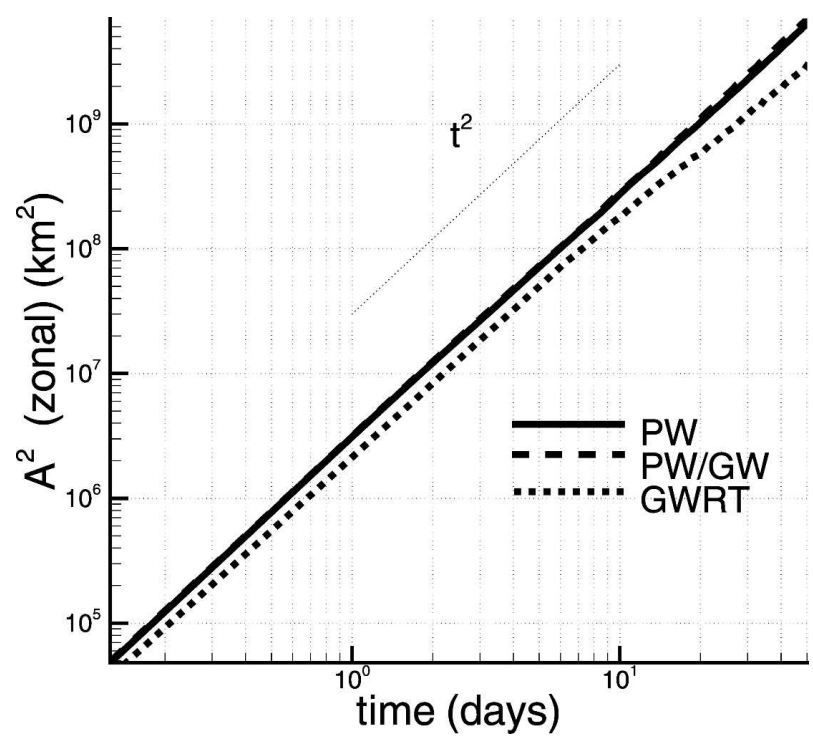

FIG. 8. Ensemble-averaged zonal absolute dispersion for the PW, PW/GW, and GWRT experiments. This and subsequent computations are performed using an ensemble of 21824 particles. Note that the offset at small $t$ is due to the fact that $A^{2}=0$ at $t=0$ so that, for the $\log -\log$ plot, the initial dispersion is at $t=3 \mathrm{~h}$ (advection time step). 
$Q_{O W} a^{2} \cos ^{2}(y) \sim 400 \mathrm{~m}^{2} \mathrm{~s}^{-2}$, which is of the same order of magnitude as the $A^{2} / t^{2}$ relation determined from Fig. 8.

Figure $7 \mathrm{~b}$ depicts the temporal autocorrelations associated with the meridional winds for the PW, PW/ GW, and GWRT experiments. The distinction between the PW and PW/GW experiments is evident in the more rapid decay of the Lagrangian correlation function for the PW/GW experiment: the presence of a finite Lagrangian time scale implies diffusive behavior in the meridional direction in the long-time limit. The presence of negative correlations, as in the case of the autocorrelations for the zonal winds, may be an artifact of oscillatory behavior induced by planetary waves. However, as previously noted, longer experimental runs would be required to verify this hypothesis.

The connection between Lagrangian autocorrelation behavior and single-particle dispersion (Taylor 1921) as described in section $3 \mathrm{~b}$ is evidenced in the ensembleaveraged absolute meridional dispersion shown in Fig. 9. In particular, the rapid decay in the autocorrelation is reflected in diffusive behavior in the latter stages of the PW/GW and GWRT experiments. A ballistic regime ( $\alpha$ $\sim 2$ ) extends to $t \sim 3$ days for the PW and PW/GW experiments, indicating that particles experience unidirectional motion until this time. Subdiffusive $(\alpha<1)$ behavior, characteristic of trapping such as observed in Levy flights (Weeks et al. 1996), is apparent after $t \sim 4$ days, when the absolute dispersion has a value of $\sim 3 \times$ $10^{5} \mathrm{~km}^{2}$, corresponding to an rms absolute displacement of approximately $500 \mathrm{~km}$. Differences between the PW and PW/GW experiments become apparent only after $t \sim 20$ days. While dispersion in the PW experiment plateaus, dispersion in the $\mathrm{PW} / \mathrm{GW}$ experiment continues to evolve subdiffusively, thus demonstrating the ability of gravity waves to enhance the displacement of individual particles. More marked is the contrast with the GWRT experiment: in that case, diffusive $(\alpha=1)$ behavior is exhibited for most of the temporal range considered. This is a consequence of the absence of a planetary wave: no coherent features are present in the meridional direction for this spatially and temporally decorrelated flow. Also, as demonstrated in the patchiness plots presented in Fig. 6, some structure is apparent for the meridional component of the flow, although less dominant than for the zonal component. These features are even less distinctive for the PW/GW experiment. One can imagine, in a manner analogous to the physical picture presented by Pasmanter (1988), that both chaotic and regular particle trajectories exist in the meridional direction, so subdiffusive rather than superdiffusive behavior might be expected. This is, indeed, found to be the case for single-particle meridional dispersion. Thus in contrast to zonal abso-

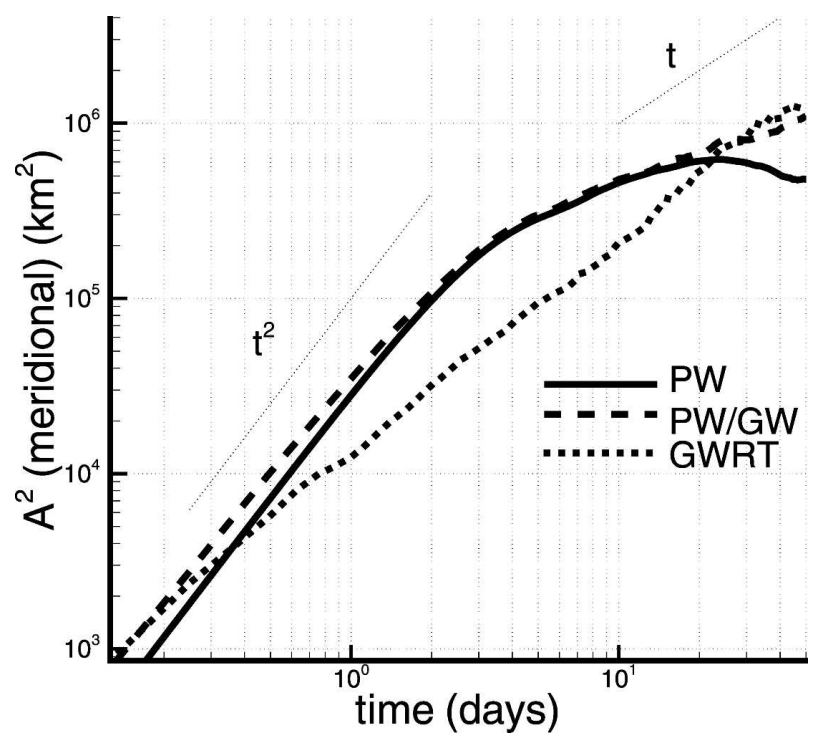

FIG. 9. Ensemble-averaged meridional absolute dispersion for the PW (solid), PW/GW (dashed), and GWRT (dotted) experiments. Note the offset at small $t$ for the same reason as described in Fig. 8.

lute dispersion, differences between the stratospheric and mesospheric regimes are apparent in the meridional absolute dispersion.

The time evolution of the zonal-displacement PDFs, expressed in terms of $\delta x=x(t)-x(0)$, (where the PDFs are normalized to have area unity with a bin size of $2200 \mathrm{~km}$ ), is shown in Fig. 10. These PDFs exhibit long tails illustrative of eastward advection by the zonal shear flow in all three experiments. Translation of particles occurs via large-scale advection, regardless of the presence or absence of gravity waves. Comparison of the PDFs for the PW and PW/GW experiments demonstrates slightly enhanced zonal dispersion for the PW/GW experiment. A compressed PDF for the GWRT experiment suggests that gravity waves, in the absence of coherent features established by the PW forcing, smear the wide range of sweeping velocities in the zonal direction. This may be explained in terms of Taylor's (1953) theory of shear-induced dispersion where it was found that molecular diffusion acts to inhibit along-shear transport for a solute of uniform concentration. Strong GWRT forcing prevents particles from remaining in the center of the westerly jet long enough to move a large distance zonally. Skewness and flatness (not shown) reveal non-Gaussian behavior, as is to be expected in the presence of a zonal shear flow and has been noted in previous stratospheric transport studies (Seo and Bowman 2000).

The effect of unbalanced dynamics on meridional dispersion is demonstrated in the PDFs for meridional 
a)

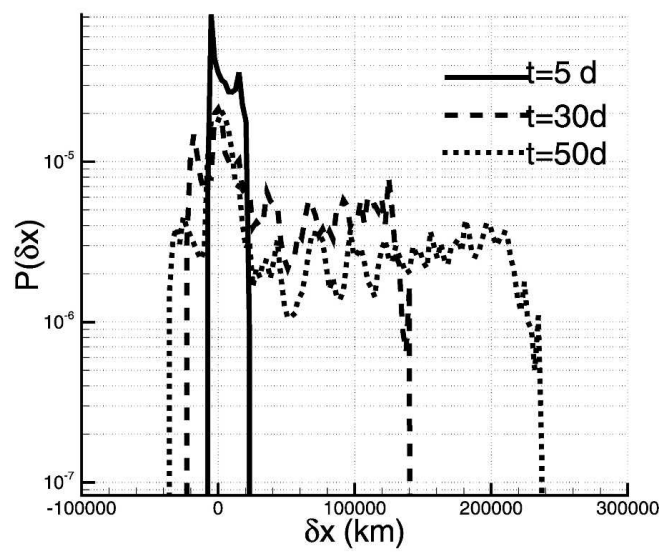

b)

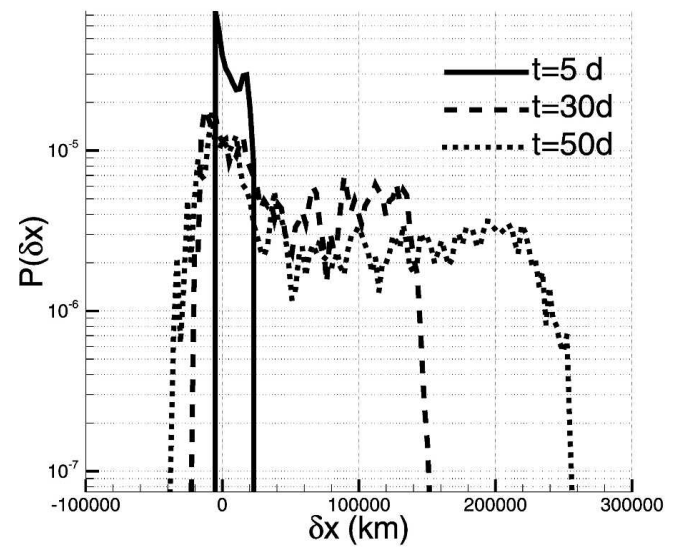

c)

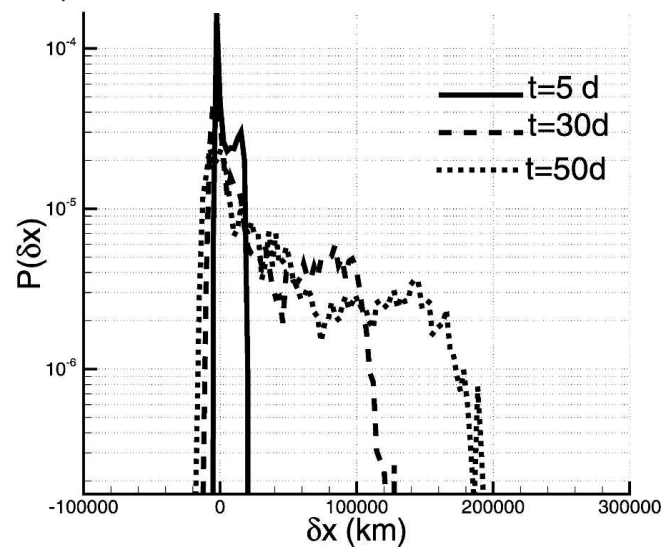

FIG. 10. Probability distribution functions for zonal absolute displacement at different instants in time for the (a) PW, (b) PW/GW, and (c) GWRT experiments.

absolute displacement expressed in terms of $\delta y=y(t)$ $-y(0)$, and depicted in Fig. 11. Here, the role of the gravity waves in establishing a more diffusive regime is evidenced in the more Gaussian PDFs for the PW/GW a)

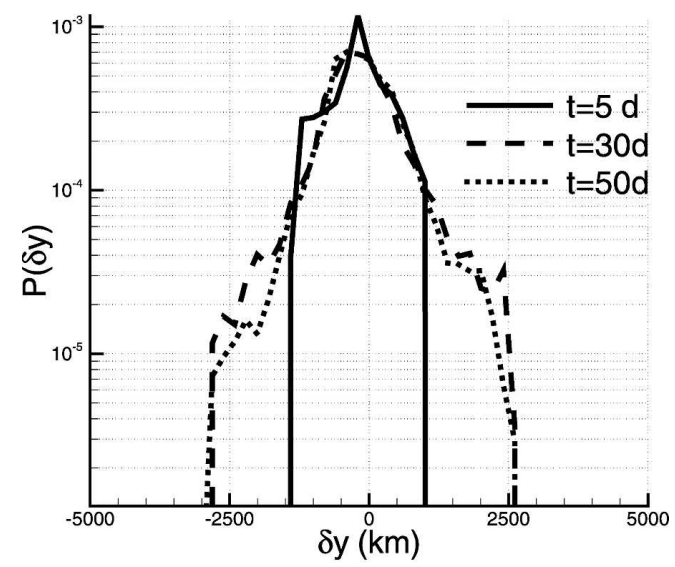

b)

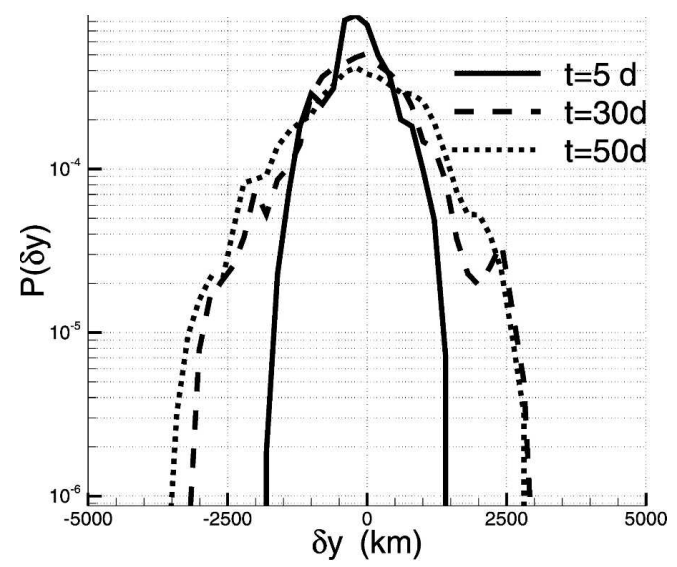

c)

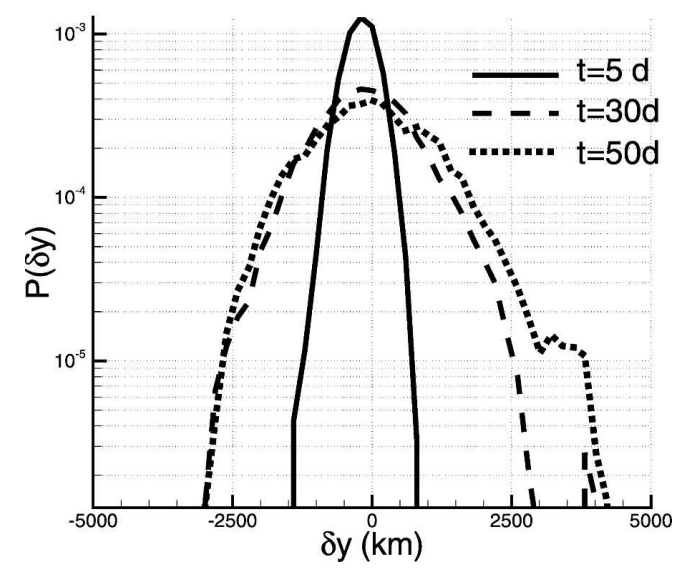

FIG. 11. As in Fig. 10 but for the meridional absolute displacement.

experiment relative to the $\mathrm{PW}$ experiment. While the skewness (not shown) is essentially zero for all three experiments-the flow is symmetric, that is, there is no preferred direction of displacement in the absence of a 
basic-state shear flow in the meridional direction-Fig. 12 shows that the flatness is close to $F=3$ for the PW/GW and GWRT experiments, both of which are signatures of the Gaussian behavior that characterizes a diffusive regime. Noteworthy also from the absolute displacement PDFs is the existence of exponential tails for the PW experiment. Often cited as a signature of large-scale intermittency (Majda and Kramer 1999), exponential as opposed to Gaussian decay is deemed a reflection of large fluctuations in passive tracer behavior. In the present case, we speculate that these fluctuations may be attributed to intermittency associated with nonlocal dynamics. Previous stratospheric analyses (Sparling and Bacmeister 2001; Majda and Kramer 1999) have also found a direct link between scale-dependent exponential functions and increment PDFs, which represent intermittency due to a separation in scales between the advecting wind field and the passive tracer field or, equivalently, nonlocal dynamics. The results for the PW/GW and GWRT experiments are, in contrast, reminiscent of the behavior predicted by the central limit theorem, namely the emergence of a Gaussian distribution in the long-time limit $\left(t \gg T_{L}\right)$, for $T_{L}$ the Lagrangian integral time scale, and thus local dynamics.

A central feature to be noted from the zonal relative dispersion statistics is the difference between results obtained for the PW and PW/GW experiments, in contrast to zonal single-particle dispersion statistics. Shown in Fig. 13 are the Lagrangian autocorrelations for the latitudinal and longitudinal gradients in the zonal $\left(\partial_{y} u\right.$ and $\partial_{x} u$, respectively) and meridional $\left(\partial_{y} v\right.$ and $\partial_{x} v$, respectively) winds. The meridional wind gradients are included in the present analysis since consideration of velocity gradients and hence vorticity, strain, and divergence in the context of relative dispersion requires consideration of the gradients in $u$ and $v$, a feature exemplified in the Hua-Klein diagnostic. Figure 13 indicates that the autocorrelations for the PW/GW and GWRT experiments decay much more rapidly than those for the PW experiment. Negative correlations may be a signature of oscillations in the $\mathrm{PW}$ experiment, or a consequence of large positive latitudinal gradients of the zonal winds (evident in Figs. 2a and 5a). The largest spatial gradients in the Lagrangian velocity are found at the edge of the zonal jet (see, e.g., the patchiness plots), hence in the latitudinal gradient of the zonal wind $\partial_{y} u$. The slow decay in the PW experiment for $\partial_{y} u$, which is the dominant term for the vorticity $\omega=\partial_{x} v-\partial_{y} u$, suggests that the PW experiment evolves on very slow time scales. By contrast, the rapid decorrelation in both $\partial_{x} u$ and $\partial_{y} v$, which comprise the divergence $\delta=\partial_{x} u+$ $\partial_{y} v$, for the $\mathrm{PW} / \mathrm{GW}$ experiment suggests that the unbalanced component dominates for $t \sim 3$ days. Studies

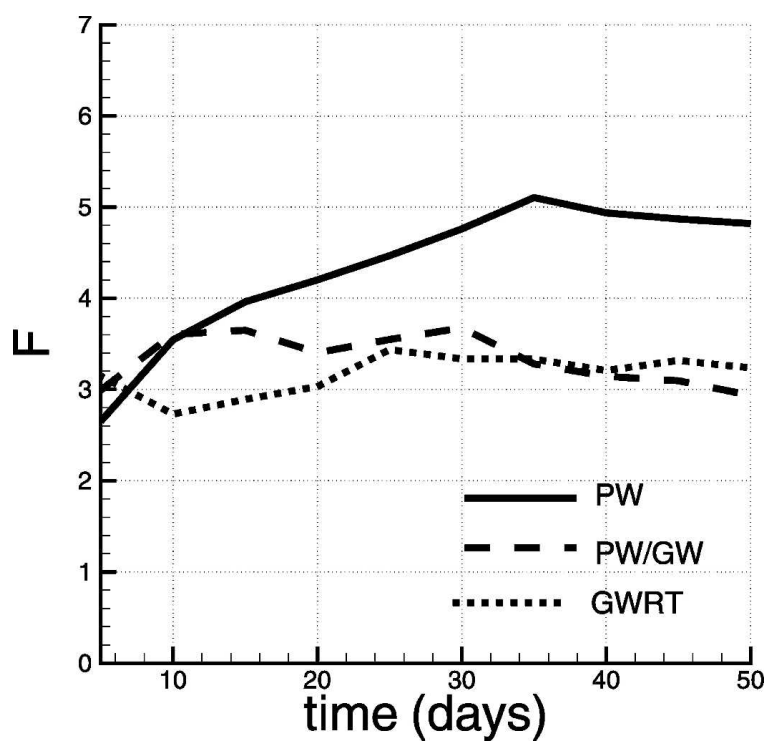

FIG. 12. Flatness of the meridional absolute displacement PDF for the PW, PW/GW, and GWRT experiments.

by Babiano et al. (1990) have demonstrated an inverse relationship between the characteristic time scale denoting the transition from short [(23)] to intermediate times and velocity gradients. The existence of such a time scale ( $\sim 3$ days) for the $\mathrm{PW} / \mathrm{GW}$ experiment suggests that the intermediate regime characterized by Richardson's (1926) $t^{3}$ law will be attained more rapidly in the presence of a divergent component (as noted in section 3c) than for the PW experiment.

The Lagrangian autocorrelation behavior is elucidated in the ensemble-averaged relative zonal dispersion $D^{2}$ versus time, depicted in Fig. 14. The dominance of advection associated with a zonal shear flow is demonstrated in the longer duration of linear relative dispersion for the PW experiment: linear dispersion characterizes short-time behavior in each of the three experiments due to the nonzero mean advective term $\langle W(t)\rangle$ relative to the quadratic term in (23). Evident in particular is the more rapid approach to Richardson's (1926) $t^{3}$ intermediate regime for the $\mathrm{PW} / \mathrm{GW}$ compared to the PW experiment, and for the GWRT compared to the $\mathrm{PW} / \mathrm{GW}$ experiment. The temporal variability associated with the gravity waves enhances separation between particle pairs, thus destroying memory of initial particle separation more rapidly than for the PW experiment. In particular, a pair of particles initially trapped within a given region may be allowed to escape. This has important implications for regions such as the surf zone and the edge of the polar vortex in the middle atmosphere: the introduction of gravity waves allows particles trapped within the core of the cat's eye structure to permeate surrounding regions, 
a)

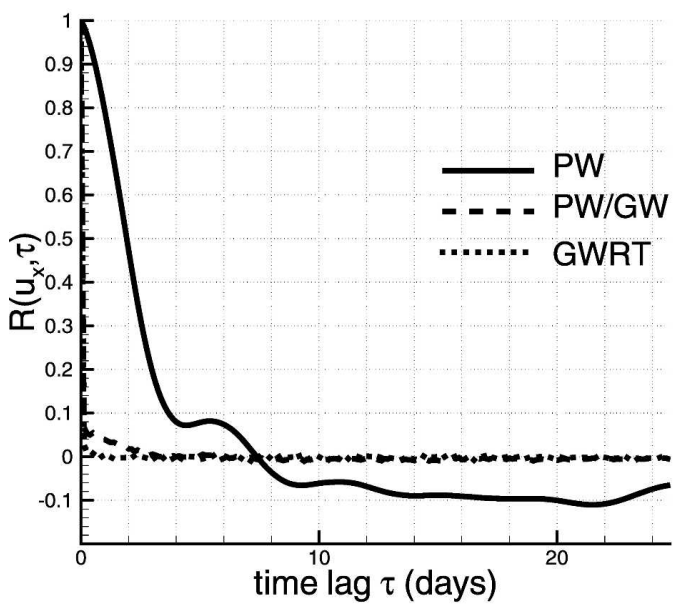

c)

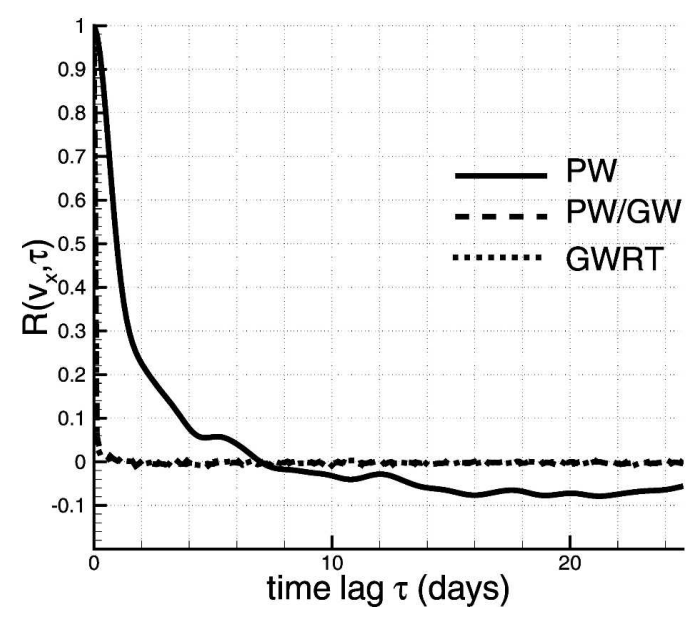

b)

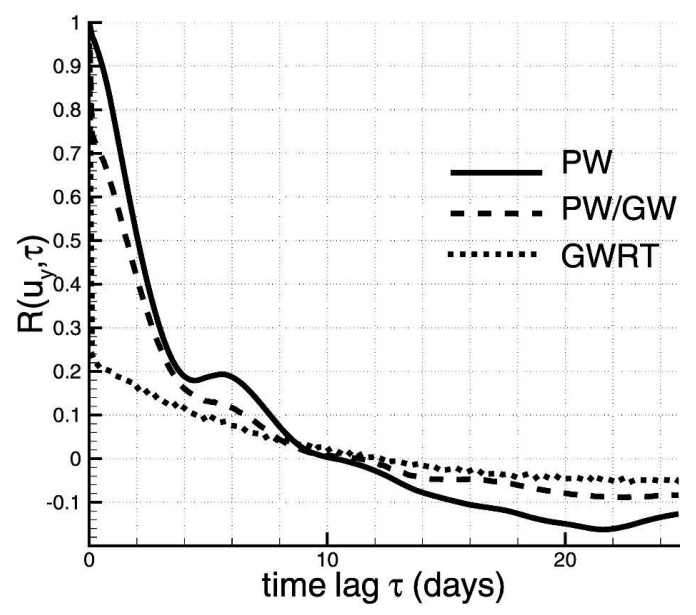

d)

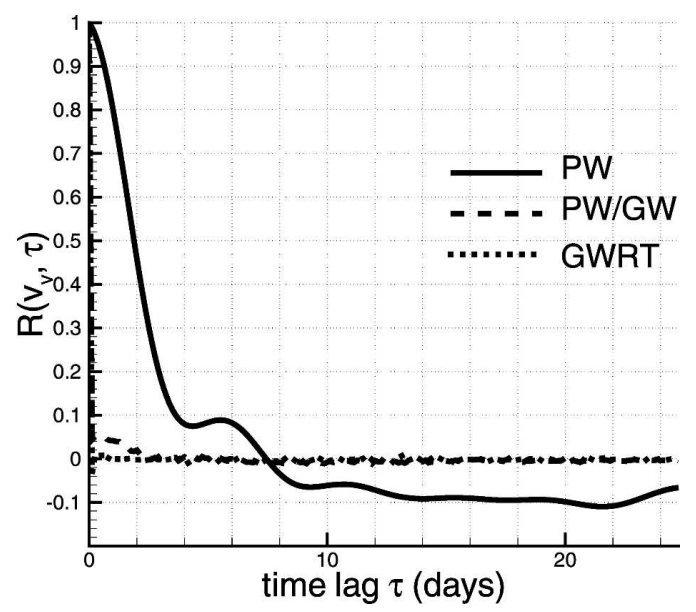

FIG. 13. Lagrangian autocorrelations for (a) $\partial_{x} u$, (b) $\partial_{y} u$, (c) $\partial_{x} v$, and (d) $\partial_{y} v$, namely, the velocity gradients.

while particles found near the edge of the polar vortex may wander into midlatitude regions. This mechanism is evident in the zonal relative dispersion statistics: the linear behavior depicted in (23) is replaced more rapidly by Richardson's $t^{3}$ regime for the PW/GW than for the PW experiment, underlining the role of the divergent component in eroding coherent structures established by planetary waves. Such behavior is further demonstrated in meridional relative dispersion statistics, where the trapping mechanism established by PWs in the meridional direction is perturbed by the GWs, allowing particles to escape the surf zone, as is discussed below. Geometrical considerations have already indicated that gravity waves destroy the coherence of the stirring mechanisms present in the PW case (see, e.g., Fig. 4). As such, gravity waves may be viewed as a catalyst for mixing, and relative dispersion the tool with which the nature of this mixing is observed.
Figure 15 depicts the ensemble-averaged meridional relative dispersion. Meridional dispersion is characterized by a quadratic $\left(t^{2}\right)$ regime for short times (on the order of hours) in the absence of a mean advective term [cf. (23)], and diffusive behavior at intermediate times, for the PW, PW/GW, and GWRT experiments. It takes longer for the diffusive $(t)$ limit to be attained for the GWRT experiment most likely due to the absence of a coherent PW structure (the surf zone), within which spatial homogeneity is more rapidly attained. In the absence of planetary waves such diffusive behavior persists for long times, as is demonstrated in the GWRT experiment. However, the introduction of organized structure to the flow with the planetary wave forcing and the suppression of the gravity waves results in trapping phenomena in the PW experiment, as shown in the plateau corresponding to a meridional trapping region of width $\sim 1000 \mathrm{~km}$ or $\sim 10^{\circ}$ latitude. This value is com- 


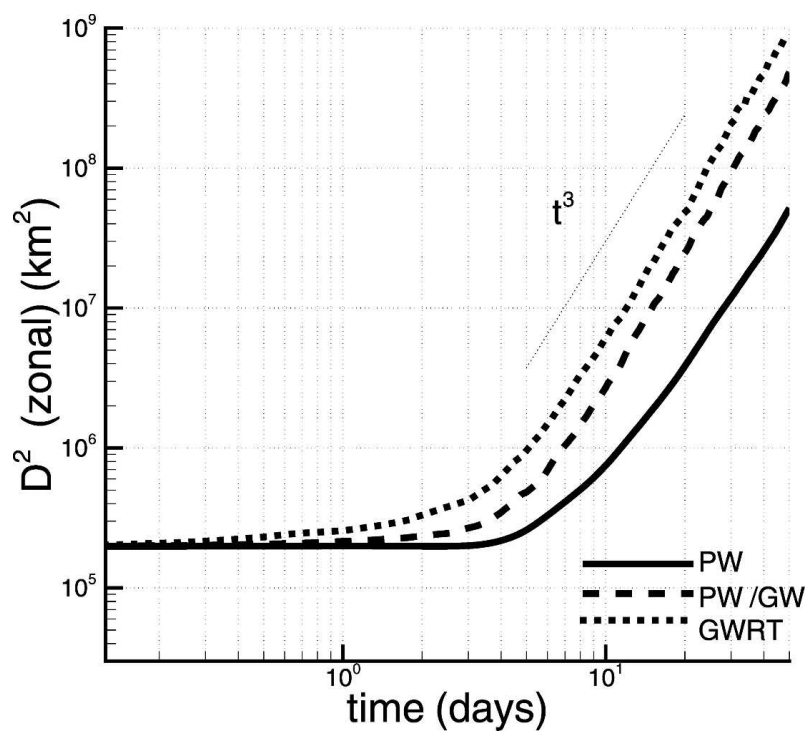

FIG. 14. Ensemble-averaged zonal relative dispersion for the PW, PW/GW, and GWRT experiments, represented by solid, dashed, and dotted lines, respectively.

parable to the meridional width of the region of strong vorticity at midlatitudes shown by the Okubo-Weiss and Hua-Klein criteria in Fig. 4. As previously noted, the combined effect of planetary and gravity waves erodes this inhomogeneity, as shown in the continued increase in the meridional relative dispersion $D^{2}$ after $t$ $\sim 20$ days for the PW/GW experiment, yet the effect of the planetary waves is still reflected in the subdiffusive behavior for $t>4$ days, in striking contrast to the GWRT experiment. Thus, only after $t>10$ days do

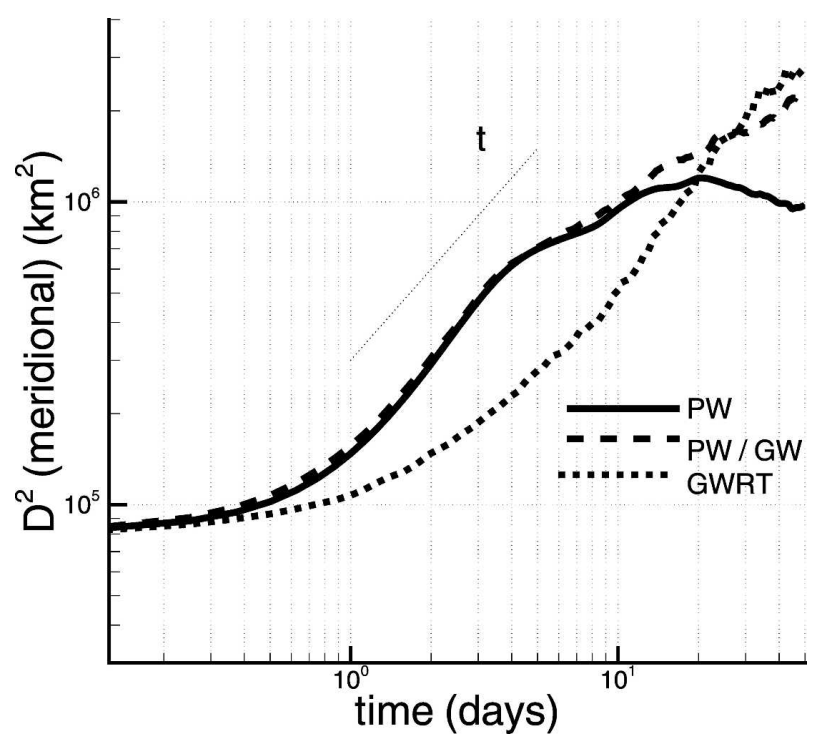

FIG. 15. As in Fig. 14 but for the meridional relative dispersion. particle pairs lose memory of their initial separation [the elapsed time will differ for different $D_{0}$; see, e.g., (23)], at which time the effects of unbalanced dynamics become apparent for the PW/GW experiment. The long-time meridional relative dispersion results are consistent with those found from the meridional absolute dispersion analysis. Both emphasize the emergence of diffusive behavior in the presence of an unbalanced component to the flow.

As with single-particle statistics, Taylor's (1953) theory of shear-induced dispersion is relevant to the case of zonal relative dispersion, in the sense that a solute initially concentrated at a single point will spread. Connected also to Taylor's theory of shearinduced dispersion is the phenomenon wherein meridional dispersion enhances zonal dispersion. The meridional separation PDFs are shown in Fig. 16. The PDFs for the PW experiment vary little in time between 30 and 50 days, which reflects the subdiffusive behavior observed in Fig. 15. The PDFs for the PW/GW and GWRT experiments reveal that unbalanced dynamics result in enhanced separation between particle pairsthe introduction of a fast component increases the range of eddies to which particle pairs are subjected, allowing particles to travel beyond the confines of the surf zone. In the case of zonal relative dispersion, shown in Fig. 17, the most narrow distribution is that associated with the PW experiment since the separation between a pair of particles is monitored, rather than the individual particle displacement. With the introduction of gravity waves, separation is enhanced in the zonal direction, thus demonstrating the ability of meridional diffusive mechanisms to enhance zonal dispersion of a particle cluster. The stretched tails evident in the PW/ GW case signal large particle displacements induced by the advection by the basic-state shear flow and planetary wave, combined with enhanced local separation resulting from diffusive processes. The difference in scales between Figs. 10 and 17 should be noted. Relative dispersion analysis indicates that particles-within the solute, for Taylor's theory, and tracer particles for the present problem-are allowed to separate more easily with a rapidly fluctuating component to the flow.

\section{c. Structure functions: Spectral nonlocality and locality}

The implications of a modified flow topology for mixing, and subsequent determination of an appropriate eddy diffusivity are illustrated in structure function scaling laws. Shown in Fig. 18a are the structure functions associated with the $\mathrm{PW}, \mathrm{PW} / \mathrm{GW}$, and GWRT experiments computed at $45^{\circ} \mathrm{N}$ and averaged over 50 days. The structure functions at other latitudes are simi- 
a)

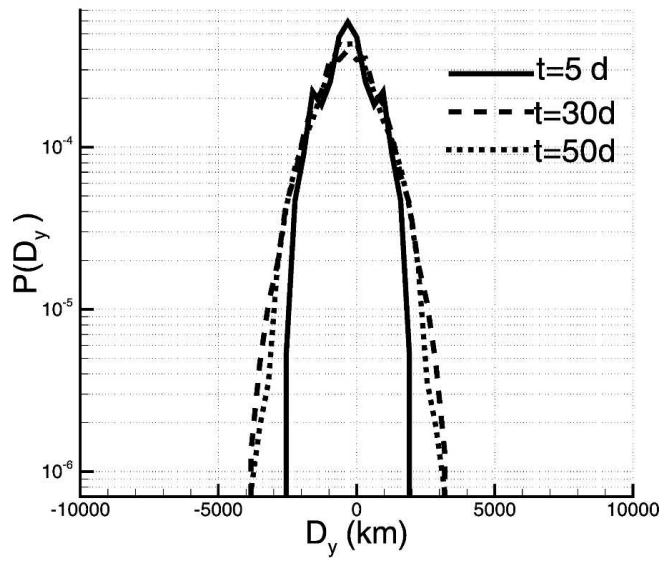

b)

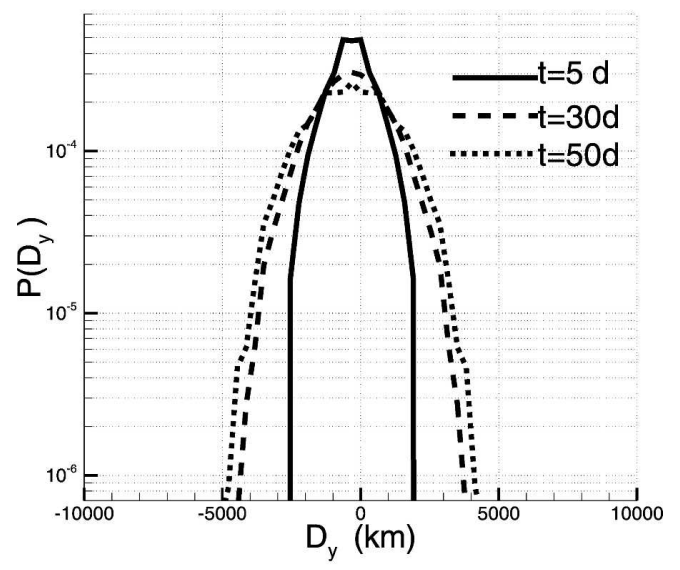

c)

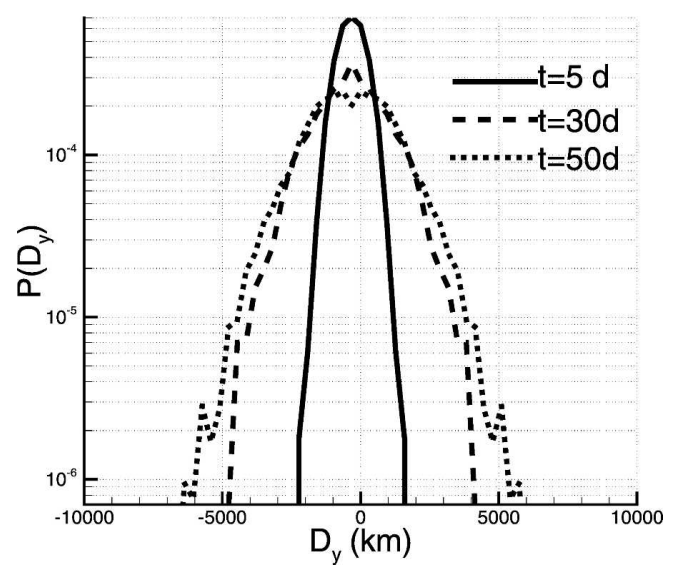

FIG. 16. PDF of the meridional separation at different instants in time for the (a) PW, (b) PW/GW, and (c) GWRT experiments.

lar. The scaling laws appropriate for nonlocal and local dynamics as derived for two-dimensional turbulence (Bennett 1984; Babiano et al. 1985) appear to be obeyed for each of the three experiments in that the a)

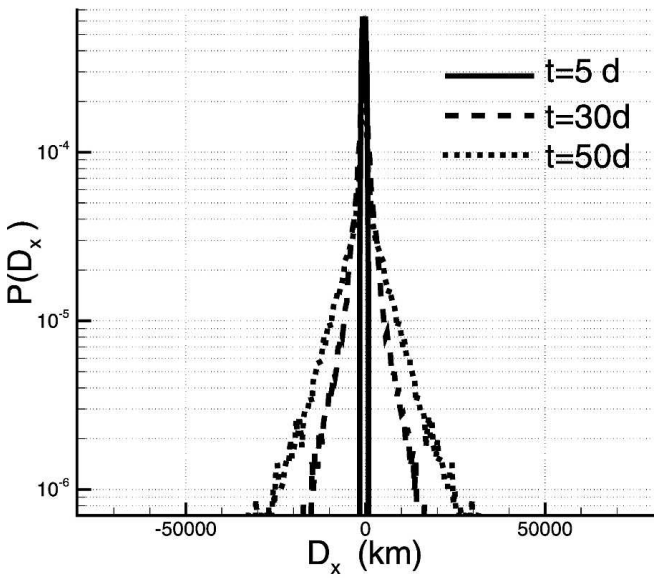

b)

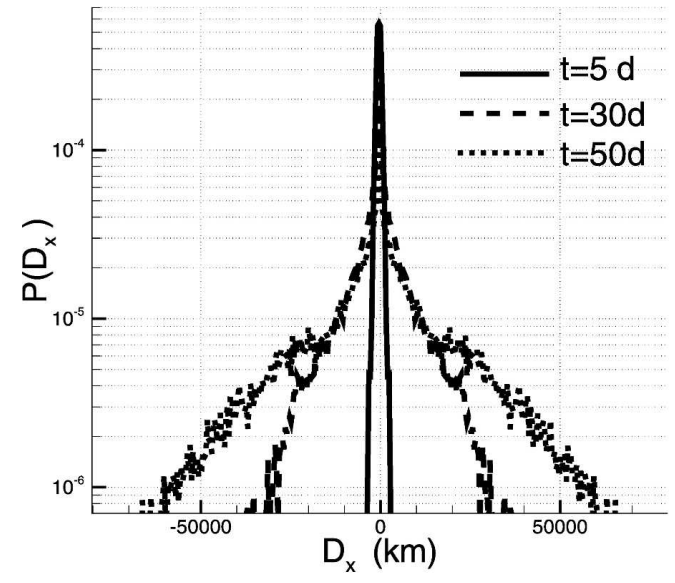

c)

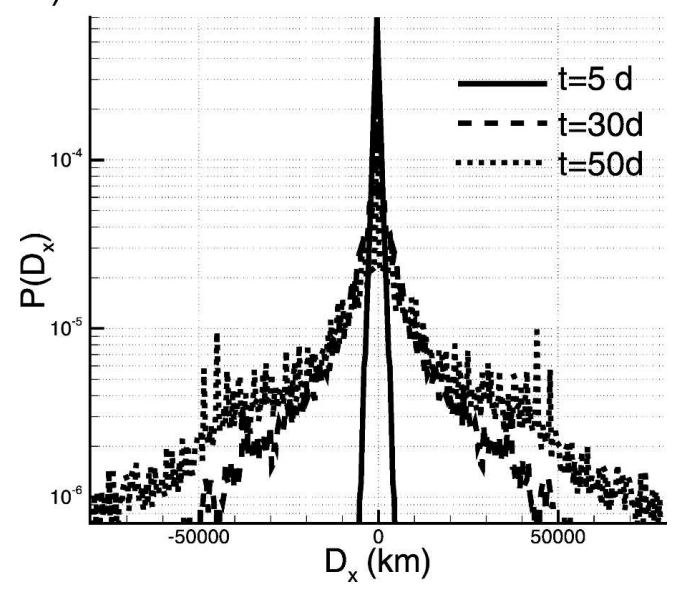

FIG. 17. As in Fig. 16 but of the zonal separation.

structure function results of Fig. 18b coincide with scaling laws established for the KE spectra shown in Fig. 18b. That is, for the case of PW forcing where nonlocal interactions are expected to dominate, a $\mathcal{D}^{2}$ scaling law 
a)

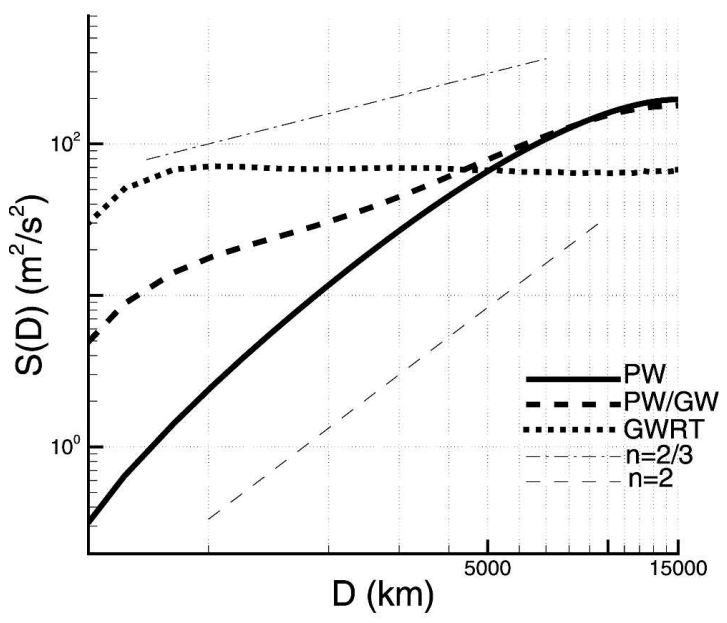

b)

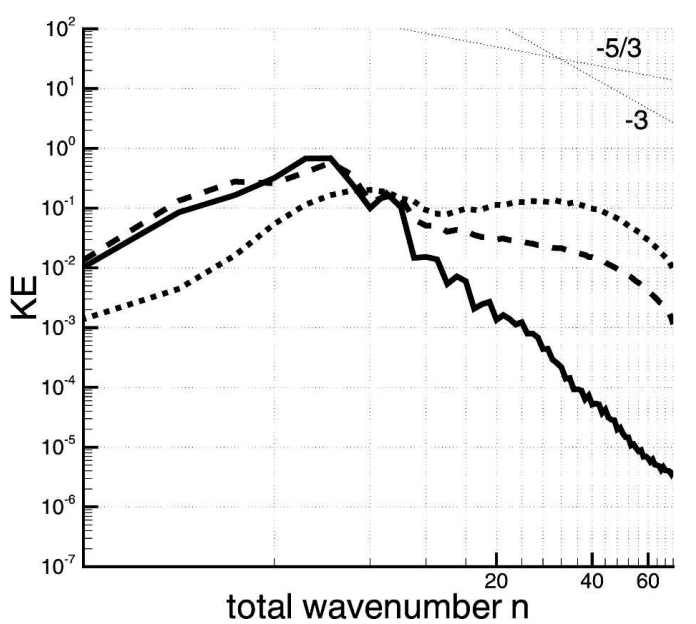

FIG. 18. Temporally averaged structure function at $45^{\circ} \mathrm{N}$ for the $\mathrm{PW}, \mathrm{PW} / \mathrm{GW}$, and GWRT experiments. Indicated also are the slopes corresponding to $n=2$ and $n=2 / 3$. (b) Corresponding kinetic energy spectra $\left(\mathrm{m}^{2} \mathrm{~s}^{-2}\right)$ with reference slopes indicated.

is obtained at all spatial scales in accordance with (2). However, with the presence of the smaller-scale eddies resulting from the $\mathrm{GW}$ forcing in the $\mathrm{PW} / \mathrm{GW}$ experiment, the structure function approaches an intermediate scaling law of $S(\mathcal{D}) \sim \mathcal{D}^{2 / 3}$. According to (3), this corresponds to $E(k) \sim k^{-5 / 3}$, which roughly approximates the kinetic energy spectrum achieved in this experiment (Fig. 18b). By contrast, the structure function approaches a constant for the GWRT experiment, as is to be expected for the diffusive regime established by the spatially random forcing. Figure 18 suggests that the scaling laws derived for 2D turbulence do indeed hold for unbalanced flow. That is, the structure-function scaling laws appear to be insensitive to the presence of a strong divergent component to the flow.
The insensitivity of 2D turbulence arguments to the introduction of a divergent component suggests that physical parameters such as the instantaneous eddy diffusivity defined in (29) may be derived from structure function scaling laws, regardless of whether the flow is governed by unbalanced or balanced dynamics.

\section{d. Eddy diffusivities}

Both the absolute and relative dispersion statistics demonstrate that the PW/GW experiment exhibits behavior similar to the PW experiment at short time scales and to the GWRT experiment over longer time scales. Evidence of Gaussian statistics in the $\sqrt{t} \mathrm{rms}$ displacement, skewness $S \sim 0$ and flatness $F \sim 3$ for the absolute dispersion analysis suggests that a constant diffusivity provides a plausible characterization of transport in the meridional direction (Fig. 9) for the $\mathrm{PW} / \mathrm{GW}$ and particularly the GWRT experiment [see, e.g., (25)]. Enhanced separation in the meridional separation PDFs in the presence of an unbalanced component to the flow further supports a diffusive description for transport in unbalanced flow. The apparent insensitivity of the structure-function scaling laws derived for 2D turbulence to the presence of an unbalanced component to the flow, as demonstrated in the structure function result $S \sim \mathcal{D}^{2 / 3}$ (see Fig. 18), suggests that unbalanced dynamics may be represented by an instantaneous separation-dependent diffusivity, defined in accordance with the model spatial resolution [see (29)]. Noteworthy also is the fact that the results from the structure function analysis for the $\mathrm{PW} / \mathrm{GW}$ experiment $\left(S \sim \mathcal{D}^{2 / 3}\right)$, corresponding to local dynamics, yield an instantaneous relative eddy diffusivity that is consistent [see (26)] with Richardson's (1926) predicted $\mathfrak{D}^{4 / 3}$ scaling.

\section{Discussion}

The present investigation has sought to elucidate the influence of an unbalanced, divergent component to the flow in the context of zonal and meridional adiabatic transport in an idealized stratosphere and mesosphere. Geometrical and statistical analyses have demonstrated that the introduction of a strong unbalanced component to the flow with the inclusion of gravity waves, as manifested in shallow slopes of kinetic energy spectra, does indeed have important implications for transport and mixing.

Geometrical considerations demonstrate that velocity gradients are strongly influenced by the presence of an unbalanced component to the flow. There is an erosion of velocity-gradient structure in the Hua-Klein di- 
agnostic in the $\mathrm{PW} / \mathrm{GW}$ experiment relative to the $\mathrm{PW}$ experiment. Zonal patchiness plots exhibit a persistence in coherent spatial structure owing to the dominant role played by the basic-state shear in the competition between the basic-state shear, PWs, and GWs. By contrast, meridional patchiness plots exhibit enhanced variability in the competition between PWs and GWs, and an erosion of spatial structure in the surf zone. Thus unbalanced dynamics are shown to induce enhanced dispersion in the meridional direction, in the absence of mean meridional flow.

Statistical analyses reinforce the geometrical results. The survival of coherent features exhibited by patchiness is confirmed by anomalous diffusion and nonGaussian PDFs obtained in single-particle zonal dispersion analyses. The effect of unbalanced dynamics on zonal transport is reflected by relative rather than absolute dispersion statistics, in particular in the more rapid approach to Richardson's (1926) $t^{3}$ regime for the $\mathrm{PW} / \mathrm{GW}$ experiment than for the PW experiment. Separation PDFs for the PW/GW and GWRT experiments demonstrate also that a rapidly fluctuating component to the flow enhances along-shear transport and zonal spread in a particle cluster, which is consistent with Taylor's (1953) theory of shear-induced dispersion. By contrast, both the single- and two-particle statistical analyses provide further evidence of more diffusive behavior in the meridional direction resulting from the inclusion of an unbalanced component to the flow. The plateau attained in meridional dispersion statistics for the PW experiment is replaced by subdiffusive behavior for the PW/GW experiment as the gravity waves act to erode the coherent surf-zone structure imposed by the planetary wave. A purely diffusive regime is established in the absence of such coherent features, as is demonstrated by the results for the GWRT experiment. The establishment of a more diffusive regime in the presence of unbalanced motion is demonstrated also in the appearance of Gaussian statistics for meridional absolute and relative dispersion PDFs associated with the PW/GW and GWRT experiments.

Spectral locality and nonlocality for the stratospheric (PW) and mesospheric (PW/GW) regimes were explored using structure function scaling laws. Largescale sweeping effects indicative of stirring are represented by the nonlocal behavior depicted in the $D^{2}$ scaling for the structure function, in a manner consistent with the balanced dynamics observed in $k^{-3}$ scaling for the kinetic energy spectra. The homogenization of small-scale structure, a signature of mixing, is represented by the local dynamics characterized by the $D^{2 / 3}$ structure function scaling for a corresponding kinetic energy spectrum with $\sim k^{-5 / 3}$ scaling indicative of un- balanced dynamics. These results further suggest that meridional transport in the mesosphere may be modeled as a diffusive process, and quantified by an instantaneous eddy diffusivity that can be determined from structure-function scaling laws.

Acknowledgments. This paper is based on the first author's Ph.D. thesis at the University of Toronto, during which time support was provided by fellowships from the Natural Sciences and Engineering Research Council of Canada, OGSST, the Department of Physics at the University of Toronto, and the Sumner Foundation. Particle advection studies were performed using code developed by K. Ngan. The numerical code used to compute the patchiness plots was provided by the Control and Dynamical Systems division of the California Institute of Technology at the "Lagrangian Transport, Stirring and Mixing in Geophysical Flows" summer school held in August 1999.

\section{REFERENCES}

Babiano, A., C. Basdevant, and R. Sadourny, 1985: Structure function and dispersion laws in two-dimensional turbulence. J. Atmos. Sci., 42, 942-949.

— _ - P. Le Roy, and R. Sadourny, 1990: Relative dispersion in two-dimensional turbulence. J. Fluid Mech., 214, 535-557.

Bacmeister, J. T., S. D. Eckermann, P. A. Newman, L. Lait, K. R. Chan, M. Loewenstein, M. H. Proffitt, and B. L. Gary, 1996: Stratospheric horizontal wavenumber spectra of winds, potential temperature, and atmospheric tracers observed by high-altitude aircraft. J. Geophys. Res., 101, 9441-9470.

Basdevant, C., and T. Philipovitch, 1994: On the validity of the "Weiss criterion" in two-dimensional turbulence. Physica D, 73, 17-30.

Batchelor, G. K., 1949: Diffusion in a field of homogeneous turbulence I. Eulerian analysis. Aust. J. Sci. Res., 2, 437-450.

_ 1951: Diffusion in a field of homogeneous turbulence II. The relative motion of particles. Proc. Camb. Philos. Soc., 48, 345-362.

Bennett, A. F., 1984: Relative dispersion: Local and nonlocal dynamics. J. Atmos. Sci., 41, 1881-1886.

Charney, J. G., and P. G. Drazin, 1961: Propagation of planetaryscale disturbances from the lower into the upper atmosphere. J. Geophys. Res., 66, 83-109.

Fritts, D. C., and M. J. Alexander, 2003: Gravity wave dynamics and effects in the middle atmosphere. Rev. Geophys., 41, 1003, doi: 10.1029/2001RG000106.

Haller, G., 2000: Finding finite-time invariant manifolds in twodimensional velocity fields. Chaos, 10, 99-108.

Hua, B. L., and P. Klein, 1998: An exact criterion for the stirring properties of nearly two-dimensional turbulence. Physica D, 113, 98-110.

Joseph, B., and B. Legras, 2002: Relation between kinematic boundaries, stirring and barriers for the Antarctic polar vortex. J. Atmos. Sci., 59, 1198-1212.

Juckes, M. N., and M. E. McIntyre, 1987: A high resolution, onelayer model of breaking planetary waves in the stratosphere. Nature, 328, 590-596. 
Koshyk, J., B. Boville, K. Hamilton, E. Manzini, and K. Shibata, 1999: Kinetic energy spectrum of horizontal motions in middle-atmosphere models. J. Geophys. Res., 104, 177-189.

LaCasce, J. H., and K. G. Speer, 1999: Lagrangian statistics in unforced barotropic flows. J. Mar. Res., 57, 245-274.

Lapeyre, G., B. L. Hua, and B. Legras, 2001: Comment on "Finding finite-time invariant manifolds in two-dimensional velocity fields." Chaos, 11, 427-430.

Louazel, S., and B. L. Hua, 2004: Vortex erosion in a shallowwater model. Phys. Fluids, 8, 3079-3085.

Majda, A. J., and P. R. Kramer, 1999: Simplified models for turbulent diffusion: Theory, numerical modelling, and physical phenomena. Phys. Rep., 314, 238-574.

Malhotra, N., I. Mezić, and S. Wiggins, 1998: Patchiness: A new diagnostic for Lagrangian trajectory analysis in timedependent fluid flow. Int. J. Bifurcation Chaos, 8, 1053-1093.

McIntyre, M., and T. Palmer, 1983: Breaking planetary waves in the stratosphere. Nature, 305, 593-600.

Ngan, K., and T. G. Shepherd, 1999a: A closer look at chaotic advection in the stratosphere. Part I: Geometric structure. $J$. Atmos. Sci., 56, 4134-4152.

_ and - 1999b: A closer look at chaotic advection in the stratosphere. Part II: Statistical diagnostics. J. Atmos. Sci., 56, 4153-4166.

Okubo, A., 1970: Horizontal dispersion of floatable particles in the vicinity of velocity singularities such as convergences. Deep-Sea Res., 17, 445-454.

Pasmanter, R., 1988: Anomalous diffusion and anomalous stretching in vortical flows. Fluid. Dyn. Res., 3, 320-326.

Pierrehumbert, R. T., 1991: Large-scale horizontal mixing in planetary atmospheres. Phys. Fluids A, 3, 1250-1260.

Polvani, L. M., and R. A. Plumb, 1992: Rossby wave breaking, microbreaking, filamentation and secondary vortex forma- tion: The dynamics of a perturbed vortex. J. Atmos. Sci., 49, 462-476.

Provenzale, A., 1999: Transport by coherent barotropic vortices Annu. Rev. Fluid Mech., 31, 55-93.

Richardson, L. F., 1926: Atmospheric diffusion shown on a distance-neighbour graph. Proc. Roy. Soc. London, A110, 709737.

Salmon, R., 1998: Lectures on Geophysical Fluid Dynamics. Oxford University Press, $378 \mathrm{pp}$.

Samelson, R., 1992: Fluid exchange across a meandering jet. $J$. Phys. Oceanogr., 22, 431-440.

Seo, H. K., and K. P. Bowman, 2000: Levy flights and anomalous diffusion in the stratosphere. J. Geophys. Res., 105, 12295 12302 .

Shepherd, T. G., 2000: The middle atmosphere. J. Atmos. SolarTerr. Phys., 62, 1587-1601.

_ J. N. Koshyk, and K. Ngan, 2000: On the nature of largescale mixing in the stratosphere and mesosphere. J. Geophys. Res., 105, 12 433-12 446.

Sparling, L. C., and J. T. Bacmeister, 2001: Scale dependence of tracer microstructure: PDFs, intermittency and the dissipation scale. Geophys. Res. Lett., 28, 2823-2826.

Taylor, G. I., 1921: Diffusion by continuous movement. Proc. London Math. Soc., 20, 196-212.

_ 1953: Dispersion of soluble matter in solvent flowing slowly through a tube. Proc. Roy. Soc. London, A219, 186-203.

Weeks, E. R., J. S. Urbach, and H. L. Swinney, 1996: Anomalous diffusion in asymmetric random walks with a quasigeostrophic flow example. Physica D, 97, 291-310.

Weiss, J., 1991: The dynamics of enstrophy transfer in twodimensional hydrodynamics. Physica D, 48, 273-294.

Zouari, N., and A. Babiano, 1994: Derivation of the relative dispersion law in the inverse energy cascade of 2-dimensional turbulence. Physica D, 76, 318-328. 